Pacific Journal of Mathematics

NTEGRALS WHICH ARE CONVEX FUNCTIONALS. II 


\title{
INTEGRALS WHICH ARE CONVEX FUNCTIONALS, II
}

\author{
R. T. ROCKAFELLAR
}

Formulas are derived in this paper for the conjugates of convex integral functionals on Banach spaces of measurable or continuous vector-valued functions. These formulas imply the weak compactness of certain convex sets of summable functions, and they thus have applications in the existence theory and duality theory for various optimization problems. They also yield formulas for the subdifferentials of integral functionals, as well as characterizations of supporting hyperplanes and normal cones.

Let $T$ be an arbitrary set, let $\mathscr{T}$ be a $\sigma$-field of subsets of $T$ (the "measurable" sets), and let $d t$ denote a nonnegative, $\sigma$-finite measure on $\mathscr{T}$. We shall be interested in functionals of the form

$$
I_{f}(u)=\int_{T} f(t, u(t)) d t, \quad u \in L,
$$

where $L$ is a linear space of measurable functions from $T$ to $R^{n}$, and $f$ is a function from $T \times R^{n}$ to $R^{1} \cup\{+\infty\}$ such that the function $f_{t}=f(t, \cdot)$ is convex on $R^{n}$ for every $t \in T$. A functional of the form $I_{f}$ is obviously convex (with values in $R^{1} \cup\{+\infty\}$ ), provided that it is well-defined in the sense that, for every $u \in L, f(t, u(t))$ is a measurable function of $t$ which majorizes at least one summable function of $t$.

In our preceding paper with the same title [16], rather general spaces $L$ were considered, but here the cases $L=L_{n}^{\infty}(T)$ and $L=L_{n}^{1}(T)$ dominate. (We denote by $L_{n}^{p}(T)=L_{n}^{p}(T, \mathscr{T}, d t), 1 \leqq p \leqq+\infty$, the Banach space consisting of all (equivalence classes of) measurable functions $u: T \rightarrow R^{n}$ such that the realvalued function $t \rightarrow|u(t)|$, where $|\cdot|$ denotes the Euclidean norm in $R^{n}$, belongs to $L^{p}(T, \mathscr{T}, d t)$ the norm of the function $t \rightarrow|u(t)|$ in $L^{p}(T, \mathscr{T}, d t)$ being the $L_{n}^{p}(T)$-norm of $u$.)

We assume as in [16] that $f$ is a normal convex integrand on $T \times R^{n}$, in other words,

(a) $f_{t}$ is for each $t$ a lower semicontinuous convex function from $R^{n}$ to $R^{1} \cup\{+\infty\}$ which is not identically $+\infty$, and

(b) there exists a countable collection $U$ of measurable functions from $T$ to $R^{n}$, such that $f(t, u(t))$ is measurable in $t$ for every $u \in U$, and $U(t) \cap D(t)$ is dense in the (nonempty, convex) set

$$
D(t)=\left\{x \in R^{n} \mid f(t, x)<+\infty\right\}
$$


for every $t \in T$, where

$$
U(t)=\{u(t) \mid u \in U\}
$$

This assumption guarantees, in particular, that $f(t, u(t))$ is a measurable function of $t$ for every measurable function $u: T \rightarrow R^{n}$ [16]. The naturalness of the class of normal convex integrands is shown by a number of characterizations in [17]. (A somewhat different approach to the measurablity question has been taken by Ioffe and Tikhomirov in [6], where $(T, \mathscr{T}, d t)$ is a Lebesgue space but $R^{n}$ is replaced by a separable Banach space. Results in [17] indicate that the two approaches are equivalent where their domains overlap.)

Condition (b) is always satisfied if $f_{t}$ is actually independent of $t$. It is equivalent, in the case where the interior of $D(t)$ is nonempty for every $t$, to the condition that $f(t, x)$ be measurable in $t$ for each $x \in R^{n}$ [16]. Condition (b) is also satisfied, of course, in the important case of a "discrete" measure space, where $d t$ assigns unit mass to each point of $T$; then the functional $I_{f}$ in (1.1) is given by a series:

$$
I_{f}(u)=\sum_{t \in T} f_{t}\left(u_{t}\right) \text {. }
$$

Let $f^{*}$ be the integrand conjugate to $f$, that is

$$
f^{*}\left(t, x^{*}\right)=\sup \left\{\left\langle x, x^{*}\right\rangle-f(t, x) \mid x \in R^{n}\right\},
$$

where $\langle\cdot, \cdot\rangle$ denotes the inner product in $R^{n}$. Then $f^{*}$ is a normal convex integrand [16], and $f$ is in turn the integrand conjugate to $f^{*}$. We have shown in [16, Theorem 2] that, if $f(t, u(t))$ is majorized by a summable function of $t$ for at least one choice of $u \in L_{n}^{p}(T)$, and similarly $f^{*}\left(t, u^{*}(t)\right)$ is majorized by a summable function of $t$ for at least one $u^{*} \in L_{n}^{q}(T)$, where $(1 / p)+(1 / q)=1$, then the convex functionals $I_{f}$ on $L_{n}^{p}(T)$ and $I_{f^{*}}$ on $L_{n}^{q}(T)$ are well-defined and conjugate to each other with respect to the pairing

$$
\left\langle u, u^{*}\right\rangle=\int_{T}\left\langle u(t), u^{*}(t)\right\rangle d t .
$$

Thus one has

$$
\begin{aligned}
& I_{f^{*}}\left(u^{*}\right)=\sup \left\{\left\langle u, u^{*}\right\rangle-I_{f}(u) \mid u \in L_{n}^{p}(T)\right\}, \quad u^{*} \in L_{n}^{q}(T), \\
& I_{f}(u)=\sup \left\{\left\langle u, u^{*}\right\rangle-I_{f^{*}}\left(u^{*}\right) \mid u^{*} \in L_{n}^{q}(T)\right\}, \quad u \in L_{n}^{p}(T) .
\end{aligned}
$$

This result leaves an open question, however, in the case of $p=\infty$, since in general $L_{n}^{1}(T)$ cannot be identified with the dual space $L_{n}^{\infty}(T)^{*}$ of $L_{n}^{\infty}(T)$.

What is the nature of the convex function $I_{f}^{*}$ on $L_{n}^{\infty}(T)^{*}$ which 
is conjugate to $I_{f}$ on $L_{n}^{\infty}(T)$ ? This question is of more importance than might be expected judging from the "obscurity" of the space $L_{n}^{\infty}(T)^{*}$.

In applying the methods of functional analysis to optimization problems, such as problems in optimal control and the calculus of variations, one often finds it convenient to work in $L_{n}^{\infty}(T)$ or one of its subspaces (e.g., a space of continuous functions), where $T$ is a real interval or a region in $R^{n}$. Many of the functionals and mappings one encounters in such cases have nicer continuity or differentiability properties with respect to the "uniform" norm on $L_{n}^{\infty}(T)$ than with respect to the other $L_{n}^{p}(T)$ norms. Unfortunately, there is also a disadvantage to working in $L_{n}^{\infty}(T)$ : it is more difficult to use arguments based on duality. This drawback is serious when dealing with convexity, since duality methods are especially natural in such situations. Thus, even if $L_{n}^{\infty}(T)^{*}$ is not a convenient space to work with directly, it can be helpful to have a formula for $I_{f}^{*}$ on $L_{n}^{\infty}(T)^{*}$ in terms of $f$, because this may facilitate reasoning based on the general theory of conjugate convex functions.

By definition, one has

$$
I_{f}^{*}(v)=\sup \left\{v(u)-I_{f}(u) \mid u \in L_{n}^{\infty}(T)\right\}, \quad v \in L_{n}^{\infty}(T)^{*},
$$

assuming that $I_{f}$ is well-defined on $L_{n}^{\infty}(T)$. Formula (1.6) (when valid) implies that $I_{f}^{*}$ reduces to $I_{f^{*}}$ on $L_{n}^{1}(T)$, if $L_{n}^{1}(T)$ is identified in the canonical way with a subspace of $L_{n}^{\infty}(T)^{*}$.

Our basic result below (Theorem 1 ) is that $I_{f}^{*}$ is the direct sum of $I_{f^{*}}$ on $L_{n}^{1}(T)$ and a certain "singular" component, which is a positively homogeneous, convex functional on the measure-theoretic complement of $L_{n}^{1}(T)$ in $L_{n}^{\infty}(T)^{*}$. We give a condition, generalizing Theorem 4 of [16], under which the "singular" component is trivial, so that $I_{f}^{*}$ is identically $+\infty$ outside of $L_{n}^{1}(T)$ and hence reduces entirely to $I_{f^{*}}$. This property implies the weak compactness in $L_{n}^{1}(T)$ of all the convex level sets of the functional $I_{f^{*}}$ (see Corollaries $2 \mathrm{~A}$ and 2B). The sufficient condition for weak compactness which is obtained in this way may be regarded as a generalization of Nagumo's Theorem [12] in the calculus of variations, and it is also related to recent work of Olech $[13,14]$ in the same area. Furthermore, it generalizes a result of Castaing [2, Lemma 7.1] as formulated by Valadier [21, 1.15]. The latter corresponds to the case where $f_{t}$ is for each $t$ the support function (and $f_{t}^{*}$ the indicator function) of a nonempty, compact, convex subset of $R^{n}$. (For extensions in this case to spaces more general than $R^{n}$, see [2], [3], and [4]). (Note added in revision: since the writing of this paper, Valadier [22, p. 14-16] has independently proved, in effect, a slightly weaker form of our compact- 
ness result, Corollary 2B, and Castaing [5, Theorem 6] has established a generalization in which $R^{n}$ is replaced by a reflexive Banach space. We have also proved a converse of Corollary $2 \mathrm{~B}$ in [20].)

After establishing these basic results in $\S 2$, we extend them in $\S 3$ to the situation where $T$ is a topological space, $L_{n}^{\infty}(T)$ is replaced by a space of continuous, $R^{n}$-valued functions on $T$, and $L_{n}^{\infty}(T)^{*}$ is correspondingly replaced by a space of $R^{n}$-valued measures on $T$. The argument is based on a general theorem about the images and inverse images of convex functions under continuous, linear transformations between locally convex, topological vector spaces.

A refinement is then given in $\S 4$ under the assumption that the multifunction $D: t \rightarrow D(t)$ in (1.2) is "fully lower semicontinuous." A theorem of Michael [9] concerning continuous selections is essential to the proof. The refined formula yields in $\S 5$ a characterization of the weak* closure of certain convex sets of measures on $T$ (Corollaries 5B, 5C) and a corresponding compactness criterion (Corollary 5D). The latter extends a lemma which Olech [13, 14] uses as the basic tool in deducing the existence of solutions to problems in optimal control.

Throughout the paper, we give applications to the theory of subgradients. If $I_{f}$ is regarded as a function on $L_{n}^{p}(T)$, the subgradients of $I_{f}$ at the point $u \in L_{n}^{p}(T)$, with respect to the pairing (1.5) between $L_{n}^{p}(T)$ and $L_{n}^{q}(T),(1 / p)+(1 / q)=1$, are by definition the elements $u^{*} \in L_{n}^{q}(T)$ such that

$$
I_{f}\left(u^{\prime}\right) \geqq I_{f}(u)+\left\langle u^{\prime}-u, u^{*}\right\rangle \text { for every } u^{\prime},
$$

or equivalently

$$
I_{f}^{*}\left(u^{*}\right)=\left\langle u, u^{*}\right\rangle-I_{f}(u) .
$$

The set of all such subgradients is denoted by $\partial I_{f}(u)$. Formulas (1.4) and (1.6) (when the latter is valid) imply at once that a function $u^{*} \in L_{n}^{q}(T)$ belongs to $\partial I_{f}(u)$ if and only if

$$
f^{*}\left(t, u^{*}(t)\right)=\left\langle u(t), u^{*}(t)\right\rangle-f(t, u(t))
$$

for almost every $t$, or in other words,

$$
u^{*}(t) \in \partial f_{t}(u(t))
$$

for almost every $t$.

If $p=\infty$, and one considers the pairing between $L_{n}^{\infty}(T)$ and $L_{n}^{\infty}(T)^{*}$, rather than $L_{n}^{1}(T)$, the subgradient set $\partial I_{f}(u)$ consists by definition of the set of linear functionals $v \in L_{n}^{\infty}(T)^{*}$ such that

$$
I_{f}^{*}(v)=v(u)-I_{f}(u) .
$$


The formula for $I_{f}^{*}$ on $L_{n}^{\infty}(T)^{*}$ yields a description of the subgradients of $I_{f}$ in the more general case. Similarly, the conjugacy formulas in $\S 3$ and $\S 4$ yield descriptions of the subgradients of integral functionals on spaces of continuous functions.

2. Basic results. Recall that, in analogy with the Lebesgue decomposition of measures, every linear functional $v$ in $L_{n}^{\infty}(T)^{*}$ can be expressed uniquely as the sum of an "absolutely continuous" component $v_{a}$ and a "singular" component $v_{s}$. The "absolutely continuous" linear functional $v_{a}$ corresponds to an element $u^{*}$ of $L_{n}^{1}(T)$, in the sense that

$$
v_{a}(u)=\int_{T}\left\langle u(t), u^{*}(t)\right\rangle d t, \quad u \in L_{n}^{\infty}(T) .
$$

On the other hand, the "singular" linear functional $v_{s}$ has the property that, given any finite, nonnegative measure $\tau$ equivalent to $d t$ and any $\varepsilon>0$, there is a measurable subset $S$ of $T$ such that $\tau(T \backslash S)<\varepsilon$, and $v_{s}(u)=0$ for every $u$ is $L_{n}^{\infty}(S)$. (Here we identify $L_{n}^{\infty}(S)$ with the subspace of $L_{n}^{\infty}(T)$ consisting of the functions which vanish almost everywhere outside of $S$ ). One has

$$
\|v\|=\left\|v_{a}\right\|+\left\|v_{s}\right\|=\int_{T}\left|u^{*}(t)\right| d t+\left\|v_{s}\right\| .
$$

The set of all "singular" functionals $v_{s}$ in $L_{n}^{\infty}(T)$ is a closed subspace which may be regarded as the measure-theoretic complement of $L_{n}^{1}(T)$.

This decomposition of $L_{n}^{\infty}(T)^{*}$ may be derived from general results concerning Riesz spaces, but it also can be obtained by representing $L_{n}^{\infty}(T)$ (via the theory of commutative Banach algebras, applied to $L_{1}^{\infty}(T)$ ) as the space of all continuous functions from $\bar{T}$ to $R^{n}$, where $\bar{T}$ is a certain (extremally disconnected) compact Hausdorff space. In the latter case, $\tau$ and the elements of $L_{n}^{\infty}(T)^{*}$ can be regarded as finite, regular Borel measures on $\bar{T}$, and the ordinary Lebesgue decomposition theorem can be invoked.

As usual, we denote by $\delta_{C}$ the indicator of a convex set $C$, that is, the convex function which vanishes on $C$ and is identically $+\infty$ outside of $C$. For $C \subset L_{n}^{\infty}(T)$, the convex function $\delta_{o}^{*}$ on $L_{n}^{\infty}(T)^{*}$ conjugate to $\delta_{C}$ is given by

$$
\delta_{o}^{*}(v)=\sup \{v(u) \mid u \in C\} .
$$

Thus $\delta_{C}^{*}$ is the support function of $C$, and $\delta_{o}^{*}$ is positively homogeneous.

TheOREM 1. Assume that $f(t, u(t))$ is majorized by a summable 
function of $t$ for at least one $u \in L_{n}^{\infty}(T)$, and that $f^{*}\left(t, u^{*}(t)\right)$ is $m a$ jorized by a summable function of $t$ for at least one $u^{*} \in L_{n}^{1}(T)$. Then $I_{f}$ is well-defined on $L_{n}^{\infty}(T)$, and the convex function $I_{f}^{*}$ on $L_{n}^{*}(T)^{*}$ conjugate to $I_{f}$ is given by

$$
I_{f}^{*}(v)=I_{f^{*}}\left(u^{*}\right)+\delta_{c}^{*}\left(v_{s}\right), \quad u \in L_{n}^{\infty}(T)^{*},
$$

where $u^{*}$ is the element of $L_{n}^{1}(T)$ giving the "absolutely continuous" component of $v, v_{s}$ is the "singular" component of $v$, and

$$
C=\left\{u \in L_{n}^{\infty}(T) \mid I_{f}(u)<+\infty\right\} \text {. }
$$

Proof. As pointed out above, the hypothesis implies by [16, Theorem 2] that $I_{f}$ and $I_{f^{*}}$ are well-defined on $L_{n}^{\infty}(T)$ and $L_{n}^{1}(T)$, respectively, and that formulas (1.6) and (1.7) hold for $p=\infty$ and $q=1$. Thus for every $u^{*} \in L_{n}^{1}(T)$ and every measurable subset $S$ of $T$ one has

$$
\begin{aligned}
\int_{S} f^{*}\left(t, u^{*}(t)\right) d t= & \sup \left\{\int _ { S } \left[\left\langle u(t), u^{*}(t)\right\rangle\right.\right. \\
& \left.-f(t, u(t))] d t \mid u \in L_{n}^{\infty}(S)\right\}
\end{aligned}
$$

by (1.6) and the definition of $f^{*}$. Fix $v \in L_{n}^{\infty}(T)^{*}$, and choose any real number $\alpha$ such that

$$
\alpha<I_{f^{*}}\left(u^{*}\right)=\int_{T} f^{*}\left(t, u^{*}(t)\right) d t
$$

where $u^{*}$ satisfies (2.1). Let $\bar{u}$ be any element of $L_{n}^{\infty}(T)$ such that $f(\cdot, \bar{u}(\cdot))$ is summable, i.e., any element of the set $C$ in (2.5), and let $\delta>0$. Let $\tau$ be any finite, nonnegative measure equivalent to $d t$. The "singularity" of $v_{s}$ implies the existence of a nondecreasing sequence of measurable sets $S_{k}$ in $T$ such that

$$
\tau\left(T \backslash S_{k}\right)>1 / k,
$$

and $v_{s}$ vanishes on $L_{n}^{\infty}\left(S_{k}\right)$. Taking $S$ to be $S_{k}$ for $k$ sufficiently large, and $S^{\prime}=T \backslash S$, we have

$$
\begin{gathered}
\int_{S} f^{*}\left(t, u^{*}(t)\right) d t<\alpha, \\
\int_{S^{\prime}}\left[\left\langle\bar{u}(t), u^{*}(t)\right\rangle-f(t, \bar{u}(t))\right] d t>-\delta .
\end{gathered}
$$

We now calculate from (1.8) and (2.6) that 


$$
\begin{aligned}
I_{f}^{*}(v)= & \sup \left\{v(u)-\int_{S} f(t, u(t)) d t-\int_{S^{\prime}} f(t, u(t)) d t \mid u \in L_{n}^{\infty}(T)\right\} \\
= & \sup \left\{v_{a}(u)-\int_{S} f(t, u(t)) d t \mid u \in L_{n}^{\infty}(S)\right\} \\
& +\sup \left\{v_{a}(u)+v_{s}(u)-\int_{S^{\prime}} f(t, u(t)) d t \mid u \in L_{n}^{\infty}\left(S^{\prime}\right)\right\} \\
= & \int_{S} f^{*}\left(t, u^{*}(t)\right) d t+\sup \left\{v_{s}(u)\right. \\
& \left.+\int_{S^{\prime}}\left[\left\langle u(t), u^{*}(t)\right\rangle-f(t, u(t))\right] d t \mid u \in L_{n}^{\infty}\left(S^{\prime}\right)\right\} .
\end{aligned}
$$

Taking $u$ to be the element of $L_{n}^{\infty}\left(S^{\prime}\right)$ which agrees with $\bar{u}$ on $S^{\prime}$ but vanishes on $S$, we see from (2.10) that

$$
\begin{aligned}
I_{f}^{*}(v) \geqq & \int_{S} f^{*}\left(t, u^{*}(t)\right) d t+v_{s}(\bar{u}) \\
& +\int_{S^{\prime}}\left[\left\langle\bar{u}(t), u^{*}(t)\right\rangle-f(t, \bar{u}(t))\right] d t,
\end{aligned}
$$

and hence by (2.8) and (2.9)

$$
I_{f}^{*}(v) \geqq \alpha+v_{s}(\bar{u})-\delta .
$$

Inasmuch as $\alpha$ was any number satisfying (2.7), and $\delta$ was any positive number, we conclude from (2.12) that

$$
I_{f}^{*}(v) \geqq I_{f *}\left(u^{*}\right)+v_{s}(\bar{u}) \text {. }
$$

Moreover, this holds for any $\bar{u} \in C$. Thus

$$
\begin{aligned}
I_{f}^{*}(v) & \geqq I_{f^{*}}\left(u^{*}\right)+\sup \left\{v_{s}(\bar{u}) \mid \bar{u} \in C\right\} \\
& =I_{f^{*}}\left(u^{*}\right)+\delta_{C}^{*}\left(v_{s}\right) .
\end{aligned}
$$

To prove the opposite inequality, we observe simply that

$$
\begin{aligned}
I_{f^{*}}(v) & =\sup _{u \in C}\left\{\left\langle u, u^{*}\right\rangle+v_{s}(u)-I_{f}(u)\right\} \\
& \leqq \sup _{u \in O}\left\{\left\langle u, u^{*}\right\rangle-I_{f}(u)\right\}+\sup _{u \in C} v_{s}(u) \\
& =I_{f^{*}}\left(u^{*}\right)+\delta_{\sigma}^{*}\left(v_{s}\right) .
\end{aligned}
$$

This establishes Theorem 1.

Corollary 1A. Assume, in addition to the hypothesis of Theorem 1, that the convex set $C$ is a cone. Then in formula (2.4) one has

$$
\begin{aligned}
I_{f}^{*}(v) & =I_{f^{*}}\left(u^{*}\right) \text { if } v_{s}(u) \leqq 0 \text { for every } u \in C, \\
& =+\infty \text { otherwise. }
\end{aligned}
$$


Proof. If $C$ is a convex cone, then the support function of $C$ is the indicator of $C^{*}$, the convex cone polar to $C$.

A noteworthy case of Corollary $1 \mathrm{~A}$ occurs when $f(t, \cdot)$ is for every $t$ the support function of a nonempty closed convex set $Q(t) \subset$ $R^{n}$. Then $C$ is a cone by the positive homogeneity of $f(t, \cdot)$. The function $f^{*}(t, \cdot)$ is the indicator of $Q(t)$, and hence $I_{f^{*}}$ on $L_{n}^{1}(T)$ is the indicator of the convex set $W$ consisting of all summable functions $u^{*}$ such that $u^{*}(t) \in Q(t)$ for almost every $t$. The hypothesis of Theorem 1 is satisfied if and only if $W \neq \varnothing$, in which event $I_{f}$ on $L_{n}^{\infty}(T)$ is the support function of $W$ (regarded as a subset of $L_{n}^{\infty}(T)^{*}$ ), so that $I_{f}^{*}$ is the indicator of the closure of $W$ in the weak topology induced on $L_{n}^{\infty}(T)^{*}$ by $L_{n}^{\infty}(T)$. Thus, if $W \neq \varnothing$, the latter closure of $W$ is by Corollary $1 \mathrm{~A}$ the direct sum of $W$ and a certain "singular cone." A different version of this result will be given in Corollaries 5B and $5 \mathrm{C}$.

Another important case of Corollary $1 \mathrm{~A}$ occurs when $C$ is all of $L_{n}^{\infty}(T)$. We shall cover this case in a slightly stronger way (by avoiding a direct summability assumption on $f^{*}$ ) in Corollary $2 \mathrm{~A}$.

COROLLARY 1B. Under the hypothesis of Theorem 1, an element $v \in L_{n}^{\infty}(T)^{*}$ belongs to the subgradient set $\partial I_{f}(u)$ if and only if (1.12) holds, where $u^{*}$ is the function in $L_{n}^{1}(T)$ corresponding to the "absolutely continuous" component $v_{a}$ of $v$ as in (2.1), and the "singular" component $v_{s}$ of $v$ attains its maximum over the convex set $C$ in (2.5) at the point $u$.

Proof. In view of formula (2.4) and the general inequalities

$$
\begin{aligned}
& I_{f}^{*}(v) \geqq v(u)-I_{f}(u), \\
& f^{*}\left(t, u^{*}(t)\right) \geqq\left\langle u(t), u^{*}(t)\right\rangle-f(t, u(t)), \\
& \delta_{c}^{*}(v) \geqq v(u) \text { if } I_{f}(u)<+\infty,
\end{aligned}
$$

which follow from the definitions, relation (1.13) holds if and only if (1.11) (or equivalently (1.12)) holds, and, at the same time, the supremum in (2.3) is attained at the given point $u$.

Theorem 2. Assume that $\bar{u} \in L_{n}^{\infty}(T)$ and $r>0$ have the property that $f(t, \bar{u}(t)+x)$ is a summable function of $t$ whenever $|x|<r, x \in R^{n}$. Then $f^{*}\left(t, u^{*}(t)\right)$ is majorized by a summable function of $t$ for at least one $u^{*} \in L_{n}^{1}(T)$, so that the hypothesis of Theorem 1 is satisfied. Moreover, in this case $I_{f}$ is continuous (in the $L_{n}^{\infty}(T)$ norm) at $u$ whenever $\|u-\bar{u}\|<r$, and in formula (2.4) one has

$$
\delta_{C}^{*}\left(v_{s}\right) \geqq v_{s}(\bar{u})+r\left\|v_{s}\right\|
$$


Proof. Let $r^{\prime}$ be any number in the open interval $(0, r)$. Let $x_{1}, \cdots, x_{m}$ be elements of $R^{n}$ with $\left|x_{i}\right|<r$, such that the convex hull $P$ of $\left\{x_{1}, \cdots, x_{m}\right\}$ includes every $x$ such that $|x| \leqq r^{\prime}$. Let

$$
k(t)=\max _{i=1, \cdots, m} f\left(t, \bar{u}(t)+x_{i}\right),
$$

Then $k$ is summable on $T$. If $w \in R^{n}$ satisfies $|w-\bar{u}(t)| \leqq r^{\prime}$ for a certain $t$, then $w-\bar{u}(t) \in P$, and there exist nonnegative real numbers $\lambda_{1}, \cdots, \lambda_{m}$ such that $\lambda_{1}+\cdots+\lambda_{m}=1$ and

$$
w=\lambda_{1}\left(\bar{u}(t)+x_{1}\right)+\cdots+\lambda_{m}\left(\bar{u}(t)+x_{m}\right) .
$$

The convexity of $f_{t}$ then implies

$$
f(t, w) \leqq \lambda_{1} f\left(t, \bar{u}(t)+x_{1}\right)+\cdots+\lambda_{m} f\left(t, \bar{u}(t)+x_{m}\right) \leqq k(t) .
$$

Thus, whenever $u \in L_{n}(T)$ satisfies $\|u-\bar{u}\| \leqq r^{\prime}$, we have

$$
f(t, u(t)) \leqq k(t) \text { for almost every } t \text {. }
$$

Now for each $t$ let $Q(t)=\partial f_{t}(\bar{u}(t))$, in other words,

$$
Q(t)=\left\{x^{*} \in R^{n} \mid f^{*}\left(t, x^{*}\right)=\left\langle\bar{u}(t), x^{*}\right\rangle-f(t, \bar{u}(t))\right\} .
$$

Since $f_{t}$ is finite on a neighborhood of $\bar{u}(t)$ in $R^{n}, Q(t)$ is nonempty compact convex set whose support function is given by

$$
\begin{aligned}
\delta_{Q(t)}^{*}(x) & =\max \left\{\left\langle x, x^{*}\right\rangle \mid x^{*} \in Q(t)\right\}=f^{\prime}(t, \bar{u}(t) ; x) \\
& =\lim _{\lambda \downarrow 0}[f(t, \bar{u}(t)+\lambda x)-f(t, \bar{u}(t))] / \lambda \\
& \leqq f(t, \bar{u}(t)+x)-f(t, \bar{u}(t))
\end{aligned}
$$

$[15, \S 23]$. Since the limit of a sequence of measurable functions is measurable, formula (2.17) implies that $\delta_{Q(t)}^{*}(x)$ is for each fixed $x$ a (finite) measurable function of $t$. It follows then from [17, Theorem 3] that the multifunction $Q: t \rightarrow Q(t)$ is measurable in the sense of Castaing [2]. According to a selection theorem of Kuratowski and Ryll-Nardzewski [8] (quoted as Corollary 1.1 in [17]), there exists a measurable function $u^{*}: T \rightarrow R^{n}$ such that $u^{*}(t) \in Q(t)$ for every $t$. In view of (2.17) and the fact that $f(t, w) \leqq k(t)$ if $|w-u(t)| \leqq r^{\prime}$, we have

$$
\left\langle u^{*}(t), x\right\rangle \leqq \delta_{Q(t)}^{*}(x) \leqq k(t)-f(t, \bar{u}(t))
$$

whenever $|x|<r^{\prime}$. Therefore

$$
r^{\prime}\left|u^{*}(t)\right|=\sup _{|x| \leqq r^{\prime}}\left|\left\langle u^{*}(t), x\right\rangle\right| \leqq k(t)-f(t, \bar{u}(t)) .
$$

Since $k$ and $f(\cdot, \bar{u}(\cdot))$ are summable, we may conclude from (2.18) that $u^{*} \in L_{n}^{1}(T)$. Furthermore, according to the definition (2.16) of 
$Q(t), u^{*}$ satisfies

$$
f^{*}\left(t, u^{*}(t)\right)=\left\langle\bar{u}(t), u^{*}(t)\right\rangle-f(t, \bar{u}(t)) .
$$

Thus $f^{*}\left(\cdot, u^{*}(\cdot)\right)$ is summable, and the hypothesis of Theorem 1 is satisfied by this $u^{*}$ and the function $\bar{u}$.

In particular, $I_{f}$ is a well-defined convex functional from $L_{n}^{\infty}(T)$ to $R^{1} \cup\{+\infty\}$. If $u \in L_{n}^{\infty}$ satisfies $\|u-\bar{u}\| \leqq r^{\prime}$, then (2.15) holds, so that $I_{f}(u) \leqq \alpha$, where

$$
\alpha=\int_{T} k(t) d t<+\infty .
$$

Therefore $I_{f}$ is bounded above on a neighborhood of $u$, if $\|u-\bar{u}\|<r^{\prime}$, and it follows that $I_{f}$ is continuous at every such $u \in L_{n}^{\infty}(T)$ [1, Chap. 2, p. 92]. Of course, $r^{\prime}$ was any number such that $0<r^{\prime}<r$, and hence we may conclude that $I_{f}$ is finite and continuous at $u$, if $\|u-\bar{u}\|<r$. In particular, $\|u-\bar{u}\|<r$ implies that $u \in C$, so that

$$
\begin{aligned}
\delta_{C}\left(v_{s}\right) & =\sup \left\{v_{s}(u) \mid u \in C\right\} \\
& \geqq \sup \left\{v_{s}(u) \mid\|u-\bar{u}\|<r\right\}=v_{s}(\bar{u})+r\left\|v_{s}\right\| .
\end{aligned}
$$

Theorem 2 is thereby proved.

The following corollary generalizes Theorem 4 of [16], where we imposed the more restrictive condition that $f(t, x)$ be (finite and) essentially bounded as a function of $t$ for every $x \in R^{n}$.

Corollary 2A. Assume that $f(t, x)$ is a summable function of $t \in T$ for every $x \in R^{n}$. Then $I_{f}$ on $L_{n}^{\infty}(T)$ and $I_{f^{*}}$ on $L_{n}^{1}(T)$ are welldefined convex functionals conjugate to each other with respect to the pairing (1.5), and $I_{f}$ is finite and continuous throughout $L_{n}^{\infty}(T)$. Furthermore, the conjugate function $I_{f}^{*}$ on $L_{n}^{\infty}(T)^{*}$ reduces to $I_{f^{*}}$ on $L_{n}^{1}(T)$, in the sense that

$$
I_{f}^{*}(v)=I_{f^{*}}\left(u^{*}\right)
$$

if $v$ is an "absolutely continuous" functional corresponding to a function $u^{*} \in L_{n}^{1}(T)$, while $I_{f}^{*}(v)=+\infty$ for every other $v$.

Proof. The hypothesis of Theorem 2 is satisfied in this case with $\bar{u}(t) \equiv 0$ and $r$ arbitrarily large.

Corollary 2B (Weak Compactness). Let $g$ be a normal convex integrand on $T \times R^{n}$ whose conjugate $g^{*}$ has the property that $g^{*}(t, y)$ is summable in $t$ for every $y \in R^{n}$. Then $I_{g}$ is a well-defined convex functional from $L_{n}^{1}(T)$ to $R^{1} \cup\{+\infty\}$, not identically $+\infty$. Moreover, for every $a \in L_{n}^{\infty}(T)$ and $\alpha \in R^{1}$ the convex level set 


$$
\left\{u \in L_{n}^{1}(T) \mid I_{g}(u)+\langle u, a\rangle+\alpha \leqq 0\right\}
$$

is weakly compact (that is, compact with respect to the weak topology induced on $L_{n}^{1}(T)$ by $\left.L_{n}^{\infty}(\mathrm{T})\right)$.

Proof. Let $f=g^{*}$, Then $g=f^{*}$, and Corollary 2A may be applied. Let $a \in L_{n}^{\infty}(T)$ and $\alpha \in R^{1}$. Since $I_{f}$ is continuous at $a$, the set

$$
\left\{v \in L_{n}^{\infty}(T)^{*} \mid I_{f}^{*}(v)+v(a)+\alpha \leqq 0\right\}
$$

is weak* compact in $L_{n}^{\infty}(T)^{*}$. According to a result proved simultaneously by J. J. Moreau [11] and the author [19]. In view of Corollary $2 \mathrm{~A}$, the set $(2.20)$ can be identified with the set (2.19). The weak topology on the space $L_{n}^{1}(T)$, regarded as a subspace of $L_{n}^{\infty}(T)^{*}$, is the restriction of the weak* topology on $L_{n}^{\infty}(T)^{*}$, and hence the set (2.19) is weakly compact in $L_{n}^{1}(T)$.

REMARK. Corollary 2B can also be derived, using special convexity arguments, from the Dunford-Pettis criterion for weak compactness in $L_{1}^{1}(T)$. This has been shown recently by Valadier [22, p. 14-16]. Still another method of proof can be based on the lemma of Olech $[13,14]$ which is discussed below (see the remark following Corollary 5D). If $T$ is a compact subset of $R^{n}$ and $d t$ is Lebesgue measure, Olech's lemma, slightly generalized, can be applied to the epigraphs $Q(t)$ of the functions $g(t, \cdot)$, and one can deduce in this way that the set (2.19) is compact relative to the weak topology on $L_{n}^{1}(T)$ induced by the elements of $L_{n}^{\infty}(T)$ corresponding to continuous on $T$. Corollary 2B itself can be obtained by an extension of this argument, if $d t$ is replaced by an equivalent finite measure, and $L_{n}^{\infty}(T)$ is identified with a space of continuous functions on a compact space $\bar{T}$ as described at the beginning of this section.

Corollary 2C. The subgradient formula given in Corollary $1 B$ is also valid if there exists a function $\bar{u} \in L_{n}^{\infty}(T)$ satisfying the hypothesis of Theorem 2. Moreover, for every such $\bar{u}$ the subgradient set $\partial I_{f}(\bar{u})$ may actually be identified with a nonempty, weakly compact subset of $L_{n}^{1}(T)$ : a functional $v \in L_{n}^{\infty}(T)^{*}$ belongs to $\partial I_{f}(\bar{u})$ if and only if the "singular" component of $v$ vanishes, and function $u^{*} \in L_{n}^{1}(T)$ corresponding to $v$ satisfies (1.12).

Proof. The first assertion follows from (2.4), just as in the proof of Corollary 1B. The second assertion is obvious from (2.13) and the fact that, since $I_{f}$ is finite and continuous at $\bar{u}$ by Theorem 2 , the set $\partial I_{f}(\bar{u})$ is nonempty and weak* compact in $L_{n}^{\infty}(T)^{*}$ [10]. 
3. Application to spaces of continuous functions. Henceforth we assume that $T$ is a $\sigma$-compact, locally compact Hausdorff space, that $\mathscr{T}$ is the $\sigma$-field of Borel sets, and that $d t$ is a (nonnegative) regular Borel measure on $\mathscr{T}$. (The theorems below, and their corollaries, remain true if $\mathscr{T}$ is replaced by its completion relative to $d t$, although the wording of the proofs then needs to be changed slightly.) We denote by $C_{n}(T)$ the space of all continuous functions $u: T \rightarrow R^{n}$ vanishing at $\infty$, under the norm

$$
\|u\|=\max \{|u(t)| \mid t \in T\} .
$$

The dual of the Banach space $C_{n}(T)$ is identified as usual with $M_{n}(T)$, the space of all finite, regular, $R^{n}$-valued Borel measures on $T$. Unless otherwise stated, the absolute continuity or singularity of a measure in $M_{n}(T)$ refers to its relationship to the measure $d t$.

Suppose $I_{f}$ is well-defined on $L_{n}^{\infty}(T)$. Then the functional

$$
J_{f}(u)=\int_{m} f(t, u(t)) d t, \quad u \in C_{n}(T),
$$

is also well-defined and, being a convex functional on $C_{n}(T)$, it has a certain conjugate on the space $M_{n}(T)$, namely the convex functional $J_{f}^{*}$ defined by

$$
J_{f}^{*}(\mu)=\sup \left\{\int_{T} u(t) d \mu-\int_{T} f(t, u(t)) d t \mid u \in C_{n}(T)\right\}, \mu \in M_{n}(T) .
$$

Our aim is to derive a formula for $J_{f}^{*}$ from the formula for $I_{f}^{*}$ on $L_{n}^{\infty}(T)^{*}$.

Let $A$ be the mapping which assigns to each function $u \in C_{n}(T)$ the corresponding equivalence class in $L_{n}^{\infty}(T)$. The adjoint of $A$ is the linear transformation $A^{*}$ which assigns to each $v \in L_{n}^{*}(T)^{*}$ the unique measure $\mu \in M_{n}(T)$ such that

$$
v(A u)=\int_{T} u d \mu, u \in C_{n}(T) .
$$

Regarding $I_{f}$ as a functional defined for equivalence classes, rather than individual functions, we have

$$
J_{f}=I_{f} A \quad \text { (composition) . }
$$

Therefore $J_{f}^{*}$ can be obtained from $I_{f}^{*}$ and $A^{*}$ by the following general result, which is a corollary of theorems proved in [18] and basic facts about conjugate functions (for the finite-dimensional case, see $[15, \S 16])$.

THeOREM 3. Let $F$ and $G$ be arbitrary, locally convex Hausdorff topological vector spaces with duals $F^{*}$ and $G^{*}$. Let $g$ be a lower 
semicontinuous convex function from $G$ to $R^{1} \cup\{+\infty\}$, and let $g^{*}$ be the conjugate of $g$ on $G^{*}$. Let $A$ be a continuous linear transformation from $F$ to $G$, and let $k=g A$. Let $A^{*} g^{*}$ be the convex function on $F^{*}$ defined by

$$
\left(A^{*} g^{*}\right)\left(y^{*}\right)=\inf \left\{g^{*}\left(z^{*}\right) \mid A^{*} z^{*}=y^{*}\right\},
$$

(where the infimum is $+\infty$ by convention if $\left\{z^{*} \mid A^{*} z^{*}=y^{*}\right\}$ is empty). If $k$ is not identically $+\infty$, the convex function $k^{*}$ on $F^{*}$ conjugate to $k$ is given by

$$
k^{*}\left(y^{*}\right)=\liminf _{y_{i}^{*} \rightarrow y^{*}}\left(A^{*} g^{*}\right)\left(y_{i}^{*}\right),
$$

where the limit is taken over all nets converging to $y^{*}$ in the weak* topology (or any other topology on $F^{*}$ compatible with the duality between $F$ and $\left.F^{*}\right)$. If there is a point in the range of $A$ at which $g$ is finite and continuous, then $k^{*}=A^{*} g^{*}$, and the infimum in (3.3) is attained for each $y^{*}$ such that the set in (3.3) is nonempty.

Proof. Since $g$ is lower semicontinuous, $g$ is in turn the conjugate of $g^{*}$, in other words

$$
g(z)=\sup \left\{z^{*}(z)-g^{*}\left(z^{*}\right) \mid z^{*} \in G^{*}\right\} .
$$

It follows from this that

$$
\begin{aligned}
g(A y) & =\sup \left\{\left(A^{*} z^{*}\right)(y)-g^{*}\left(z^{*}\right) \mid z^{*} \in G^{*}\right\} \\
& =\sup \left\{y^{*}(y)-\left(A^{*} g^{*}\right)\left(y^{*}\right) \mid y^{*} \in F^{*}\right\} .
\end{aligned}
$$

Therefore the function $k=g A$ is the conjugate on $F$ of $A^{*} g^{*}$ on $F^{*}$. If $k$ is not identically $+\infty$, we may conclude from the fundamental theory of conjugate convex functions [10] that $k^{*}$ is the lower semicontinuous hull of $A^{*} g^{*}$ so that $k^{*}$ is given by (3.4). If $g$ is finite and continuous at some point of the range of $A$, we obtain the formula

$$
\begin{aligned}
k^{*}\left(y^{*}\right) & =\sup \left\{y^{*}(y)-g(A y) \mid y \in F\right\} \\
& =\min \left\{g^{*}\left(z^{*}\right) \mid z^{*} \in G^{*}, A^{*} z^{*}=y^{*}\right\}
\end{aligned}
$$

from the special case of Theorems 1 and 3 of [18] in which the function $f$ in those theorems is taken to be the linear functional $y^{*}$.

COROLLARY 3A. Under the hypothesis of Theorem 3, if there is a point of the range of $A$ at which $g$ is finite and continuous, then for every $y \in F$ the subgradient set $\partial k(y) \subset F^{*}$ is the image of the subgradient set $\partial g(A y)$ in $G^{*}$ under the transformation $A^{*}$.

Proof. The inequalities 


$$
\begin{aligned}
& k^{*}\left(y^{*}\right) \geqq y^{*}(y)-k(y), \\
& g^{*}\left(z^{*}\right) \geqq z^{*}(z)-g(z),
\end{aligned}
$$

hold by definition, with equality if and only if $y^{*} \in \partial k(y)$ and $z^{*} \in \partial g(z)$. On the other hand, here we have

$$
\begin{aligned}
k(y) & =g(A y), \\
k^{*}\left(y^{*}\right) & =\min \left\{g^{*}\left(z^{*}\right) \mid z^{*} \in G^{*}, A^{*} z^{*}=y^{*}\right\} .
\end{aligned}
$$

Thus $y^{*}$ belongs to $\partial k(y)$ if and only if there exists a $z^{*} \in G^{*}$ such that $A^{*} z^{*}=y^{*}$ and

$$
g^{*}\left(z^{*}\right)=\left(A^{*} z^{*}\right)(y)-g(A y)=z^{*}(A y)-g(A y),
$$

that is, $z^{*} \in \partial g(A y)$.

Theorem 4. Assume that $\bar{u} \in C_{n}(T)$ and $r>0$ have the property that $f(t, \bar{u}(t)+x)$ is a summable function of $t$ whenever $|x|<r$, $x \in R^{n}$. Then $J_{f}$ is a well-defined, lower semicontinuous, convex functional from $C_{n}(T)$ to $R^{1} \cup\{+\infty\}$, and $J_{f}$ is finite and continuous at every $u \in C_{n}(T)$ such that $\|u-\bar{u}\|<r$. Furthermore, $J_{f}^{*}$ on $M_{n}(T)$ satisfies

$$
\begin{aligned}
J_{f}^{*}(\mu) & =\min \left\{I_{f^{*}}\left(d \mu^{\prime} / d t\right)+\delta_{E}^{*}\left(\mu-\mu^{\prime}\right) \mid \mu^{\prime} \in M_{n}(T) \text { abso. contin. }\right\} \\
& \geqq \int_{T} \bar{u} d \mu-J_{f}(\bar{u})+r\|\nu\|,
\end{aligned}
$$

where $\nu$ is the singular component of $\mu$ and

$$
E=\left\{u \in C_{n}(T) \mid J_{f}(u)<+\infty\right\} .
$$

Proof. The first assertion is clear from Theorem 2. ( $I_{f}$ is lower semicontinuous on $L_{n}^{\infty}(T)$, since (1.7) holds for $p=\infty$ and $q=1$ ). To prove (3.5), we apply Theorem 3 with $F=C_{n}(T), G=L_{n}^{\infty}(T)$, and $A$ taken to be the canonical mapping from $C_{n}(T)$ to $L_{n}^{\infty}(T)$ described at the beginning of this section. This yields the fact that

$$
\begin{aligned}
J_{f}^{*}(\mu) & =\left(I_{f} A\right)^{*}(\mu)=A^{*} I_{f}^{*}(\mu) \\
& =\min \left\{I_{f}^{*}(v) \mid v \in L_{n}^{\infty}(T)^{*}, A^{*} v=\mu\right\} .
\end{aligned}
$$

Using the formula (2.4) for $I_{f}^{*}$, we can rewrite the last expression as

$$
\min \left\{I_{f^{*}}\left(u^{*}\right)+\delta_{\sigma}^{*}\left(v_{s}\right) \mid v \in L_{n}^{\infty}(T), A^{*} v=\mu\right\},
$$

where $u^{*} \in L_{n}^{1}(T)$ corresponds to $v_{a}$. Of course $A^{*} v_{a}=\mu^{\prime}$, where $\mu^{\prime}$ is the absolutely continuous measure in $C_{n}(T)$ with $d \mu^{\prime} / d t=u^{*}$. Thus

$$
J_{f}^{*}(\mu)=\min \left\{I_{f^{*}}\left(d \mu^{\prime} / d t\right)+K\left(\mu-\mu^{\prime}\right) \mid \mu^{\prime} \in C_{n}(T) \text { abso. contin. }\right\},
$$


where

$$
K\left(\mu-\mu^{\prime}\right)=\min \left\{\delta_{C}^{*}(w) \mid w \in L_{n}^{\infty}(T)^{*} \text { singular, } A^{*} w=\mu-\mu^{\prime}\right\} .
$$

Here the singularity restriction on $w$ can be omitted without affecting the value of $K$; this follows from (3.2) and the fact that for any $u \in C_{n}(T)$, any absolutely continuous $\mu^{\prime} \in M_{n}(T)$, and any $w \in L_{n}^{\infty}(T)^{*}$ with $A^{*} w=\mu-\mu^{\prime}$, we have

$$
\begin{aligned}
\int_{T} u(t) d \mu-\int_{T} f(t, u(t)) d t & =\left\langle A u, d \mu^{\prime} \mid d t\right\rangle-I_{f}(A u)+w(A u) \\
& \leqq I_{f^{*}}\left(d \mu^{\prime} / d t\right)+\delta_{\sigma}^{*}(w) .
\end{aligned}
$$

We now apply Theorem 3 again, but with $I_{f}$ replaced by the indicator function $\delta_{C}$. Our hypothesis implies that $A \bar{u}$ is an interior point of $C$, so that $\delta_{C}$ is finite and continuous at a point belonging to the range of $A$. Since $\delta_{E}(u)=\delta_{C}(A u)$, we therefore have

$$
\begin{aligned}
\delta_{E}^{*}\left(\mu-\mu^{\prime}\right) & =\left(\delta_{C} A\right)^{*}\left(\mu-\mu^{\prime}\right)=\left(A^{*} \delta_{C}^{*}\right)\left(\mu-\mu^{\prime}\right) \\
& =\min \left\{\delta_{\sigma}^{*}(w) \mid w \in L_{n}^{\infty}(T)^{*}, A^{*} w=\mu-\mu^{\prime}\right\}=K\left(\mu-\mu^{\prime}\right) .
\end{aligned}
$$

Thus $\delta_{E}^{*}$ can be substituted for $K$ in (3.8), and this establishes the desired equality (3.5).

To get the inequality in (3.5), we note first that the inequality

$$
I_{f^{*}}\left(d \mu^{\prime} / d t\right) \geqq \int_{T}\left\langle\bar{u}, d \mu^{\prime} / d t\right\rangle d t-\int_{T} f(t, \bar{u}(t)) d t
$$

holds for any absolutely continuous $\mu^{\prime} \in M_{n}(T)$. On the other hand, we have

$$
\delta_{E}^{*}\left(\mu-\mu^{\prime}\right) \geqq \int_{T} \bar{u} d\left(\mu-\mu^{\prime}\right)+r\left\|\mu-\mu^{\prime}\right\|,
$$

because $u \in E$ whenever $u \in C_{n}(T)$ and $\|u-\bar{u}\| \leqq r$. Adding these inequalities, we see that

$$
I_{f^{*}}\left(d \mu^{\prime} / d t\right)+\delta_{E}^{*}\left(\mu-\mu^{\prime}\right) \geqq \int_{T} \bar{u} d \mu-J_{f}(\bar{u})+r\left\|\mu-\mu^{\prime}\right\|
$$

for every absolutely continuous $\mu^{\prime}$. The inequality in (3.5) follows from the equation in (3.5) when both sides of (3.9) are minimized with respect to $\mu^{\prime}$.

Corollary 4A. Assume that $f(t, x)$ is a summable function of $t$ for every $x \in R^{n}$. Then the functional $J_{f}$ on $C_{n}(T)$ is well-defined, finite, continuous and convex. The conjugate functional $J_{f}^{*}$ on $M_{n}(T)$ is given by 


$$
J_{f}^{*}(\mu)=\int_{T} f^{*}(t, d \mu / d t) d t
$$

if $\mu \in M_{n}(T)$ is absolutely continuous, whereas $J_{f}^{*}(\mu)=+\infty$ if $\mu$ is not absolutely continuous.

Proof. The hypothesis of Theorem 4 is satisfied for $\bar{u}(t) \equiv 0$ and every $r>0$. Then $\delta_{E}^{*}\left(\mu-\mu^{\prime}\right)$ is $+\infty$ if $\mu^{\prime} \neq \mu$ and 0 if $\mu^{\prime}=\mu$.

COROLlaRY 4B. Under the hypothesis of Theorem 4, a measure $\mu \in M_{n}(T)$ belongs to the subgradient set $\partial J_{f}(u)$, where $u \in C_{n}(T)$, if and only if there exists an absolutely continuous measure $\mu^{\prime} \in C_{n}(T)$ satisfying

$$
\frac{d u^{\prime}}{d t}(t) \in \partial f_{t}(u(t)) \text { for almost every } t,
$$

such that the linear functional on $C_{n}(T)$ corresponding to $\mu-\mu^{\prime}$ attains its maximum over the set $E$ at $u$.

In fact, if $u$ has the property that the function $f(t, u(t)+x)$ is summable in $t$ for every $x$ in some neighborhood of 0 in $R^{n}$, then the set $\partial J_{f}(u)$ is nonempty and weak* compact in $M_{n}(T)$, and it consists of all the absolutely continuous measures $\mu$ satisfying

$$
\frac{d u}{d t}(t) \in \partial f_{t}(u(t)) \text { for almost every } t .
$$

Proof. This is obtained from formula (3.5) by an imitation of the proofs of Corollaries $1 \mathrm{~B}$ and $2 \mathrm{C}$.

ExAMPLE. Let $T$ be compact (and hence of finite measure), and let $\Phi$ be the continuous, real-valued, convex functional on $C_{1}(T)$ defined by

$$
\Phi(u)=\log \int_{T} e^{u(t)} d t, \quad u \in C_{1}(T) .
$$

The convexity of $\Phi$ is clear from the fact that

$$
\Phi(u)=-1+\min \left\{J_{f}(u+\alpha)-\alpha \mid \alpha \in R^{1}\right\},
$$

where $f$ is the (normal) convex integrand on $T \times R^{1}$ given by

$$
f(t, x)=e^{x} .
$$

What is the functional on $M_{1}(T)$ conjugate to $\Phi$ ? From (3.13) and the definition of the conjugate functional $\Phi^{*}$, we have 


$$
\begin{aligned}
\Phi^{*}(\mu) & =\sup _{u \in C_{1}(T)}\left\{\int_{T} u d \mu-\Phi(u)\right\} \\
& =1+\sup _{u \in C_{1}(T)} \sup _{\alpha \in R^{1}}\left\{\int_{T} u d \mu-J_{f}(u+\alpha)+\alpha\right\} \\
& =1+\sup _{\alpha \in R^{1}} \sup _{u^{\prime} \in C_{1}(T)}\left\{\int_{T}\left(u^{\prime}-\alpha\right) d \mu-J_{f}\left(u^{\prime}\right)+\alpha\right\} \\
& =1+\sup _{\alpha \in R^{1}} \alpha[1-\mu(T)]+\sup _{u^{\prime} \in C_{1}(T)}\left\{\int_{T} u^{\prime} d \mu-J_{f}\left(u^{\prime}\right)\right\},
\end{aligned}
$$

and consequently

$$
\begin{aligned}
\Phi^{*}(\mu) & =1+J_{f}^{*}(\mu) \text { if } \mu(T)=1, \\
& =+\infty \text { if } \mu(T) \neq 1 .
\end{aligned}
$$

We now calculate $J_{f}^{*}$ from Corollary $4 \mathrm{~A}$. The integrand conjugate to $f$ is given by

$$
\begin{aligned}
f^{*}\left(t, x^{*}\right) & =x^{*}\left(\log x^{*}-1\right) \text { if } x^{*}>0 \\
& =0 \text { if } x^{*}=0 \\
& =+\infty \text { if } x^{*}<0 .
\end{aligned}
$$

Therefore $J_{f}^{*}(\mu)=+\infty$, unless $\mu$ is nonnegative and absolutely continuous, in which case

$$
\begin{aligned}
J_{f}^{*}(\mu) & =\int_{T} p(t)[\log p(t)-1] d t \\
& =\int_{T} p(t) \log p(t) d t-\mu(T),
\end{aligned}
$$

where $p=d \mu / d t(0 \log 0$ interpreted as 0$)$. It follows that

$$
\begin{aligned}
\Phi^{*}(\mu) & =\int_{T} p(t) \log p(t) d t \text { if } \mu \in P, p=d \mu / d t, \\
& =+\infty \text { if } \mu \notin P,
\end{aligned}
$$

where $P$ is the set of all absolutely continuous probability measures in $M_{1}(T)$. The integral

$$
\int_{T} p(t) \log [1 / p(t)] d t=-\int_{T} p(t) \log p(t) d t,
$$

where $p$ is a probability density, plays an important role, of course, in information theory and statistical mechanics.

We note, incidentally, that the set of probability density functions $p$ satisfying

$$
\int_{T} p(t) \log [1 / p(t] d t \geqq \alpha
$$


is for each real number $\alpha$ a weakly compact convex subset of $L_{1}^{1}(T)$. This is seen by taking $g$ in Corollary $2 \mathrm{~B}$ to be the normal convex integrand $f^{*}$ in (3.16).

REMARK. Theorem 3 and Corollary $3 \mathrm{~A}$ can also be used to derive from the results in $\S 2$ various theorems concerning the "continuous" infimal convolution of convex functions, as studied by Ioffe and Tikhomirov [6, 7] and Valadier [22]. In this case one takes $F=R^{n}$ and $G=L_{n}^{\infty}(T)$, and $A$ is the mapping which assigns to each $x \in R^{n}$ the corresponding constant function $u(t) \equiv x$ in $L_{n}^{\infty}(T)$. Then $I_{f} A=$ $\varphi$, where

$$
\varphi(x)=\int_{T} f(t, x) d t, \quad x \in R^{n} .
$$

4. Integral representation of the singular component. The formula for $J_{f}^{*}$ in Theorem 4 can be improved in cases where a special expression is available for $\delta_{E}^{*}$. When the multifunction

$$
D: t \rightarrow D(t)=\left\{x \in R^{n} \mid f(t, x)<+\infty\right\}
$$

is suitably well-behaved (see below), such an expression can be given in terms of integrals of the recession function of $f^{*}$. The latter is by definition the function $h$ on $T \times R^{n}$ such that

$$
h(t, w)=\lim _{\lambda \rightarrow+\infty}\left[f^{*}\left(t, x^{*}+\lambda w\right)-f^{*}\left(t, x^{*}\right)\right] / \lambda,
$$

whenever $x^{*} \in R^{n}$ satisfies $f^{*}\left(t, x^{*}\right)<+\infty$. The fact that $f^{*}(t, \cdot)$ is a lower semicontinuous, convex function, not identically $+\infty$, implies that $h(t, \cdot)$ is a well-defined, lower semicontinuous, positively homogeneous, convex function from $R^{n}$ to $R^{1} \cup\{+\infty\}$, vanishing at 0 [15, $\S 8$ ]. Indeed $h(t, \cdot)$ is the support function of $D(t)$ :

$$
h(t, w)=\delta_{D(t)}^{*}(w)=\sup \{\langle x, w\rangle \mid x \in D(t)\}
$$

[15, Theorem 13.3].

LEMma 1. The recession function $h$ of $f^{*}$ is a normal convex integrand on $T \times R^{n}$.

Proof. Since $f$ is a normal convex integrand, the multifunction

$$
t \rightarrow G(t)=\{x, \alpha) \mid f(t, x) \leqq \alpha<\infty\}
$$

is measurable from $t$ to $R^{n+1}$ [17, Theorem 3], and hence there exists a countable collection of measurable functions 


$$
t \rightarrow\left(x_{i}(t), \alpha_{i}(t)\right), \quad i \in I,
$$

such that $G(t)$ is the closure of

$$
\left\{\left(x_{i}(t), \alpha_{i}(t)\right) \mid i \in I\right\}
$$

for each $t$ [17, Theorem 1]. Then the set $\left\{x_{i}(t) \mid i \in I\right\}$ is a dense subset of $D(t)$ for each $t$. From (3.11) we have

$$
h(t, w)=\sup _{i \in I}\left\langle x_{i}(t), w\right\rangle,
$$

and this implies the normality of $h[17$, Theorem 4].

COROLlARY. If there exists a bounded, measurable function $u$ such that $u(t) \in D(t)$ for every $t$, then the integral

$$
\int_{T} h(t, w(t)) d \theta
$$

is well-defined for every nonnegative measure $\theta \in M_{1}(T)$ and every measurable function $w: T \rightarrow R^{n}$ summable with respect to $\theta$.

Proof. One has $h(t, 0) \equiv 0$ and $h^{*}(t, u(t)) \equiv 0$, so that the integral $I_{h}$ on the $L_{n}^{1}$ space associated with $\theta$ is well-defined by [16, Theorem 2].

To state the main result of this section, we recall that the multifunction $D: t \rightarrow D(t)$ is said to be lower semicontinuous from $T$ to $R^{n}$ if, whenever $U$ is an open subset of $R^{n}$ and $t_{0}$ is an element of $T$ such that $D\left(t_{0}\right) \cap U \neq \varnothing$, there exists a neighborhood $V$ of $t_{0}$ such that $D(t) \cap U \neq \varnothing$ for every $t \in V$. We shall say that $D$ is fully lower semicontinuous if $D$ is lower semicontinuous and, in addition, one has $x_{0} \in \mathrm{cl} D\left(t_{0}\right)$ whenever there are neighborhoods $U$ and $V$ of $x_{0}$ and $t_{0}$ such that the set $\{t \in V \mid D(t) \supset U\}$ is dense in $V$. If the multifunction $t \rightarrow \operatorname{cl} D(t)$ is upper semicontinuous (that is, the set $\{(t, x) \mid x \in \operatorname{cl} D(t)\}$ is closed in $\left.T \times R^{n}\right)$, lower semicontinuity of $D$ implies full lower semicontinuity. In particular, $D$ is fully lower semicontinuous if $\operatorname{cl} D(t)$ is a fixed set independent of $t$.

THEOREM 5. Assume that $T$ is a compact space with no nonempty open sets of measure zero, and that the multifunction $D: t \rightarrow D(t)$ is fully lower semicontinuous, with int $D(t) \neq \varnothing$ for every $t$. Assume further that

$$
\int_{V}|f(t, x)| d t<+\infty
$$

whenever $V$ is an open subset of $T$ and $x$ is a point of $R^{n}$ having a neighborhood $U$ such that $U \subset D(t)$ for all $t \in V$. 
Then $J_{f}$ is a well-defined, lower semicontinuous, convex functional from $C_{n}(T)$ to $R^{1} \cup\{+\infty\}$. The convex set $E$ in (3.6) has a nonempty interior consisting of the functions $u \in C_{n}(T)$ such that

$$
u(t) \in \operatorname{int} D(t) \text { for all } t \in T,
$$

and $J_{f}$ is continuous on this interior. Furthermore, $J_{f}^{*}$ on $M_{n}(T)$ is given by the formula

$$
J_{f}^{*}(\mu)=\int_{T} f^{*}(t, d \mu / d t) d t+\int_{T} h(t, d \nu / d \theta) d \theta,
$$

where $h$ is the recession function of $f^{*}, \nu$ is the singular component of with respect to $d t$, and $\theta$ is any nonnegative measure in $M_{1}(T)$ with respect to which $\nu$ is absolutely continuous.

The proof of Theorem 5 uses the following characterizations of lower semicontinuity and full lower semicontinuity, as well as an auxilliary representation theorem, stated below as Theorem 6 .

Lemma 2. Let $D: t \rightarrow D(t) \subset R^{n}$ be a multifunction such that $D(t)$ is for each $t$ a convex set with int $D(t) \neq \varnothing$, and let

$$
G=\{(t, x) \mid x \in \operatorname{int} D(t)\} \subset T \times R^{n} .
$$

(a) $D$ is lower semicontinuous if and only if $G=\operatorname{int} G$;

(b) $D$ is fully lower semicontinuous if and only if $G=\operatorname{int} \operatorname{cl} G$.

Proof. (a) Suppose that $D$ is lower semicontinuous, and let $x_{0} \in \operatorname{int} D\left(t_{0}\right)$. It is possible to choose open subsets $U_{i}$ of $D\left(t_{0}\right), i=$ $1, \cdots, m$, such that the set

$$
U=\operatorname{int} \bigcap_{x_{i} \in U_{i}} \operatorname{co}\left\{x_{1}, \cdots, x_{m}\right\}
$$

is a neighborhood of $x_{0}$. Since $D$ is lower semicontinuous, there are neighborhoods $V_{i}$ of $t_{0}$ such that $D(t) \cap U_{i} \neq Q$ for $t \in V_{i}$. Let $V=$ $V_{1} \cap \cdots \cap V_{m}$. For $t \in V$ we can find points $x_{i} \in D(t) \cap U_{i}$ for $i=$ $1, \cdots, m$, and then

$$
D(t) \supset \operatorname{co}\left\{x_{1}, \cdots, x_{m}\right\} \supset U,
$$

the first inclusion holding because $D(t)$ is convex. Thus $V \times U$ is a neighborhood of $\left(t_{0}, x_{0}\right)$ in $G$, and we may conclude that $G$ is open.

Conversely, suppose that $G$ is open. Let $U$ be an open set such that $D\left(t_{0}\right) \cap U \neq \varnothing$. Since $D\left(t_{0}\right)$ is convex and has a nonempty interior, there is a point $x_{0}$ in $\left[\operatorname{int} D\left(t_{0}\right)\right] \cap U$. The open set $G \cap[T \times U]$ contains $\left(t_{0}, x_{0}\right)$, and hence it contains $\left(t, x_{0}\right)$ for all $t$ in some neighborhood of $t_{0}$. Thus there is a neighborhood $V$ of $t_{0}$ such that $D(t) \cap U \neq \varnothing$ 
for every $t \in V$. This shows that $D$ is lower semicontinuous.

(b) Suppose that $D$ is fully lower semicontinuous. Then $G=\operatorname{int} G$ by (a), so that to prove $G=\operatorname{int} \operatorname{cl} G$ it suffices to prove that $G \supset \operatorname{int} \operatorname{cl} G$. Let $\left(t_{0}, x_{0}\right) \in \operatorname{int} \operatorname{cl} G$. We can find points $z_{1}, \cdots, z_{m}$ in $R^{n}$ such that

$$
\begin{aligned}
& x_{0} \in \operatorname{int} \operatorname{co}\left\{z_{1}, \cdots, z_{m}\right\}, \\
& \left(t_{0}, z_{i}\right) \in \operatorname{int} \operatorname{cl} G \text { for all } i .
\end{aligned}
$$

Then we can choose open neighborhoods $U_{i}$ of $z_{i}$ and $V$ of $t$ such that $V \times U_{i} \subset \operatorname{cl} G$ and the set $U$ in (4.6) is a neighborhood of $x_{0}$. The set

$$
V_{i}=\left\{t \mid D(t) \cap U_{i} \neq \varnothing\right\}
$$

is dense in $V$ because $V \times U_{i} \subset \operatorname{cl} G$, and $V_{i}$ is also open by the lower semicontinuity of $D$. Since the intersection of a finite family of open dense subsets of $V$ is again dense in $V$, the set

$$
V^{\prime}=\left\{t \in T \mid D(t) \cap U_{i} \neq \varnothing \text { for } i=1, \cdots, m\right\}
$$

is dense in $V$. Of course $D(t) \supset U$ for $t \in V^{\prime}$, as already argued in (a). Thus $V \times U$ is an open set such that $\{t \in V \mid D(t) \supset U\}$ is dense in $V$, and it follows from the definition of full lower semicontinuity that $x_{1} \in \operatorname{cl} D\left(t_{1}\right)$ for every $x_{1} \in U$ and $t_{1} \in V$. In particular $U \subset \operatorname{cl} D\left(t_{0}\right)$, and since $D\left(t_{0}\right)$ is convex this implies $x_{0} \in \operatorname{int} D\left(t_{0}\right)$, i.e., $\left(t_{0}, x_{0}\right) \in G$.

We assume now that $G=\operatorname{int} \mathrm{cl} G$, and we prove from this that $D$ is fully lower semicontinuous. Our assumption implies of course that $G$ is open. Therefore $D$ is lower semicontinuous by (a). Let $\left(t_{0}, x_{0}\right)$ have an open neighborhood $V \times U$ such that the set $\{t \in V \mid D(t) \supset U\}$ is dense in $V$. Then $V \times U \subset \operatorname{cl} G$, so that $V \times U \subset \operatorname{int} \operatorname{cl} G=G$. Thus $\left(t_{0}, x_{0}\right) \in G$ and $a$ fortiori $x_{0} \in \operatorname{cl} D\left(t_{0}\right)$. Therefore $D$ is fully lower semicontinuous.

Proof of Theorem 5. First we show that there exist functions $u \in C_{n}(t)$ satisfying (4.3). Suppose that $t_{0}$ is any point of $T$, and that $x_{0} \in$ int $D\left(t_{0}\right)$. The multifunction $t \rightarrow \operatorname{cl} D(t)$ is lower semicontinuous, since $D$ is, and hence by E. Michael's theorem on continuous selections [9, Theorem 3.2] there is a function $u \in C_{n}(T)$ such that $u\left(t_{0}\right)=x_{0}$ and

$$
u(t) \in \operatorname{cl} D(t) \text { for all } t \in T \text {. }
$$

Then for some neighborhood $V$ of $t_{0}$ we have

$$
u(t) \in \operatorname{int} D(t) \text { for all } t \in V
$$

by Lemma 2(a). Thus $T$ can be covered by a collection of open sets $V$, for each of which there is a function $u \in C_{n}(T)$ satisfying (4.7) and (4.8). The compactness of $T$ allows us to extract a finite subcovering $V_{1}, \cdots, V_{m}$. Let 


$$
u=(1 / m)\left(u_{1}+\cdots+u_{m}\right),
$$

where $u_{i} \in C_{n}(T)$ satisfies (4.7) and (4.8) for $V_{i}$. For each $t$ the points $u_{i}(t)$ all belong to $\mathrm{cl} D(t)$, and at least one belongs to int $D(t)$. From the convexity of $D(t)$ we therefore have $u(t) \in \operatorname{int} D(t)$, so that $u$ satisfies (4.3).

We demonstrate now that, if $u$ satisfies (4.3), the function $f(t, u(t))$ is summable in $t \in T$. Let $t_{0}$ be any point of $T$. Since $u\left(t_{0}\right) \in \operatorname{int} D\left(t_{0}\right)$ and $D$ is lower semicontinuous, there exist by Lemma 2(a) a neighborhood $V$ of $t_{0}$ and an $r>0$ such that $\left[u\left(t_{0}\right)+x\right] \in \operatorname{int} D(t)$ whenever $t \in V$ and $|x|<r$. Then, according to the hypothesis of the theorem, $f\left(t, u\left(t_{0}\right)+x\right)$ is summable in $t \in V$ whenever $|x|<r$. Let $S$ be a closed neighborhood of $t_{0}, S \subset V$, such that $\left|u(t)-u\left(t_{0}\right)\right|<r$ for $t \in S$, such a neighborhood exists because $T$ is compact and $u$ is continuous. The hypothesis of Theorem 4 is satisfied with $T$ replaced by $S$ and $\bar{u}(t) \equiv u\left(t_{0}\right)$, and from the fact that $\|u-\bar{u}\|<r$ in $C_{n}(S)$ we may conclude that $f(t, u(t))$ is summable in $t \in S$. Thus each point $t_{0}$ of $T$ has a neighborhood $S$ over which the function $t \rightarrow f(t, u(t))$ is summable. $T$ can be covered by a finite number of such neighborhoods, because of compactness, and therefore $f(t, u(t))$ is summable in $t \in T$ as claimed.

Observe next that each $u \in C_{n}(T)$ satisfying (4.3) actually possesses the stronger property that, for some $r>0$, one has

$$
[u(t)+x] \in \operatorname{int} D(t) \text { for all } x \text { such that }|x|<r .
$$

This follows from Lemma 2(a). In this event $f(t, u(t)+x)$ is summable in $t \in T$ for $|x|<r$, as we have just proved. Thus the hypothesis of Theorem 4 is satisfied if the $\bar{u}$ in that hypothesis is taken to be any function satisfying (4.3). In this way we obtain the fact that $J_{f}$ is a well-defined, lower semicontinuous convex functional from $C_{n}(T)$ to $R^{1} \cup\{+\infty\}$ such that $J_{f}$ is finite and continuous at each element $u$ of $C_{n}(T)$ satisfying (4.3).

Our next step is to show that the functions satisfying (4.3) constitute the interior of the set $E$ in (3.6). They are certainly contained in int $E$ by the above. On the other hand, let $\bar{u}$ be a function int $E$. Then there is an $r>0$ such that $f(t, u(t))$ is summable in $t \in T$ for every $u \in C_{n}(T)$ with $\|u-\bar{u}\|<r$. Fix any $t_{0} \in T$. Let $x_{1}, \cdots, x_{m}$ be elements of $R^{n}$ such that $\left|x_{i}\right|<r$ and

$$
\bar{u}\left(t_{0}\right) \in \operatorname{int} \operatorname{co}\left\{\bar{u}\left(t_{0}\right)+x_{1}, \cdots, \bar{u}\left(t_{0}\right)+x_{m}\right\} .
$$

Since $\bar{u}$ is continuous, there is a neighborhood $V$ of $t_{0}$ such that the set

$$
U=\operatorname{int} \bigcap_{t \in V} \operatorname{co}\left\{\bar{u}(t)+x_{1}, \cdots, \bar{u}(t)+x_{m}\right\},
$$


is a neighborhood of $\bar{u}\left(t_{0}\right)$. We have $f\left(t, \bar{u}(t)+x_{i}\right)$ summable in $t \in T$ because $\left\|\left(\bar{u}+x_{i}\right)-\bar{u}\right\|<r$, so that $f\left(t, \bar{u}(t)+x_{i}\right)$ is finite for almost every $t$. Thus the set

$$
\left\{t \in V \mid \bar{u}(t)+x_{i} \in D(t) \text { for } i=1, \cdots, m\right\}
$$

has complement of measure zero in $V$. In view of our hypothesis that $T$ contains no nonempty open sets of measure zero, the set (4.12) must be dense in $V$. Of course, if $t$ belongs to (4.12) we have

$$
D(t) \supset \operatorname{co}\left\{\bar{u}(t)+x_{1}, \cdots, \bar{u}(t)+x_{m}\right\} \supset U .
$$

Thus the set $\{t \in V \mid D(t) \supset U\}$ is dense in $V$. Since $V \times U$ is a neighborhood of $\left(t_{0}, \bar{u}\left(t_{0}\right)\right)$, this implies that $\bar{u}\left(t_{0}\right) \in \operatorname{int} D\left(t_{0}\right)$, because $D$ is fully lower semicontinuous. Here $t_{0}$ was any point of $T$, so it follows that $\bar{u}$ satisfies (4.3).

Using these facts, we now show that the desired formula (4.4) for $J_{f}^{*}$ can be reduced to a more special representation result, which we establish separately below as Theorem 6 . Let

$$
K=\left\{u \in C_{n}(T) \mid u(t) \in \operatorname{cl} D(t), \forall t \in T\right\} .
$$

Clearly $u$ belongs to int $K$ if and only if $u$ satisfies (4.3), and thus, by what we have just proved, int $E=\operatorname{int} K$. Therefore $\delta_{E}^{*}=\delta_{K}^{*}$, and the formula in Theorem 4 gives us

$$
J_{f}^{*}(\mu)=\min \left\{I_{f^{\star}}\left(d \mu^{\prime} / d t\right)+\delta_{K}^{*}\left(\mu-\mu^{\prime}\right) \mid \mu^{\prime} \in M_{n}(T) \text { abso. contin. }\right\}
$$

Theorem 6 furnishes an integral representation of $\delta_{\mathrm{K}}^{*}$ which can be substituted in (4.13):

$$
\delta_{K}^{*}\left(\mu-\mu^{\prime}\right)=\int_{T} \delta_{\hat{Q}(t)}^{*}\left(d\left(\mu-\mu^{\prime}\right) / d \theta^{\prime}\right) d \theta^{\prime},
$$

where $Q(t)=\operatorname{cl} D(t)$, and $\theta^{\prime}$ is any nonnegative measure in $M_{1}(T)$ with respect to which $\mu-\mu^{\prime}$ is absolutely continuous. Since $\mu^{\prime}$ is absolutely continuous with respect to $d t$, we can take $d \theta^{\prime}$ be of the form $d t+d \theta$, where $\theta$ is an arbitrary, singular, nonnegative measure in $M_{1}(T)$ with respect to which the singular part $\nu$ of $\mu$ is absolutely continuous. By virtue of $(4.2)$ and the fact that $\delta_{D(t)}^{*}=\delta_{Q(t)}^{*}$, the right side of (4.14) then becomes

$$
\int_{T} h\left(t, d \mu / d t-d \mu^{\prime} / d t\right) d t+\int_{T} h(t, d \nu / d \theta) d \theta .
$$

The second integral here actually remains unchanged if $\theta$ is replaced by a measure which is not singular. Thus if $\theta$ is an arbitrary, nonnegative measure in $M_{1}(T)$ with respect to which $\nu$ is absolutely continuous, the expression (4.15) gives $\delta_{K}^{*}\left(\mu-\mu^{\prime}\right)$. Substituting this 
in (4.13), we see that

$$
\begin{aligned}
J_{f}^{*}(\mu)= & \min \left\{I_{f^{*}}\left(u^{*}\right)+I_{h}\left(d \mu / d t-u^{*}\right) \mid u^{*} \in L_{n}^{1}(T)\right\} \\
& +\int_{T} h(t, d \nu / d \theta) d \theta
\end{aligned}
$$

The proof of formula (4.4) is completed by the observation that

$$
\begin{aligned}
f^{*}\left(t, u^{*}(t)\right) & +h\left(t, d \mu / d t-u^{*}(t)\right) \\
& \geqq f^{*}\left(t, u^{*}(t)\right)+\left(d \mu / d t-u^{*}(t)\right)=f^{*}\left(t, u^{*}(t)\right),
\end{aligned}
$$

since $h$ is the recession function of $f_{t}^{*}[15$, Corollary 8.5.1], and hence

$$
I_{f^{*}}\left(u^{*}\right)+I_{h}\left(d \mu / d t-u^{*}\right) \geqq I_{f^{*}}(d \mu / d t)
$$

for every $u^{*} \in L_{n}^{1}(T)$.

EXAMPLE. Let $\nu$ be an atomic measure in $M_{n}(T)$ assigning "weights" $a_{i} \in R^{n}$ to the points $t_{i}$ for $i \in I$ (a finite or countable index set). Under the hypothesis of Theorem 5 , if the points $t_{i}$ have measure zero with respect to $d t$, one has

$$
J_{f}^{*}(\nu)=-\inf \left\{J_{f}(u) \mid u \in C_{n}(T)\right\}+\sum_{i \in I} h\left(t_{i}, a_{i}\right) .
$$

This is seen, for instance, by taking $\theta$ to be a probability measure in $M_{1}(T)$ such that $\theta\left(t_{i}\right)>0$ for every $i \in I$. Since $\nu$ is singular with respect to $t$, one has

$$
J_{f}^{*}(\nu)=\int_{T} f^{*}(t, 0) d t+\sum_{i \in I} h\left(t_{i}, \theta\left(t_{i}\right)^{-1} a_{i}\right) \theta\left(t_{i}\right)
$$

by formula (4.4). On the other hand, one has

$$
\int_{T} f^{*}(t, 0) d t=J_{f}^{*}(0)=-\inf \left\{J_{i}(u) \mid u \in C_{n}(T)\right\}
$$

by (4.4) and the definition of $J_{f}^{*}$, while

$$
h\left(t_{i}, \theta\left(t_{i}\right)^{-1} a_{i}\right)=\theta\left(t_{i}\right)^{-1} h\left(t_{i}, a_{i}\right)
$$

by the positive homogeneity of the recession function $h$.

We proceed now to establish the auxilliary representation theorem that was employed in the proof of Theorem 5 .

Theorem 6. Assume $T$ is compact. Let $Q: T \rightarrow R^{n}$ be a lower semicontinuous multifunction such that $Q(t)$ is for every $t$ a nonempty, closed convex set. Let $f_{t}=f(t, \cdot)$ be the indicator of $Q(t)$, so that $f_{t}^{*}$ is the support function of $Q(t)$, and let

$$
K=\left\{u \in C_{n}(T) \mid u(t) \in Q(t), \forall t \in T\right\} .
$$


Then $K$ is a nonempty closed convex subset of $C_{n}(T), f$ and $f^{*}$ are normal convex integrands on $T \times R^{n}$ and the support function of $K$ on $M_{n}(T)$ is given by the formula

$$
\delta_{K}^{*}(\mu)=\int_{T} f^{*}(t, d \mu / d \theta) d \theta, \quad \mu \in M_{n}(T),
$$

where $\theta$ is any nonnegative measure in $M_{1}(T)$ with respect to which $\mu$ is absolutely continuous.

Proof. It is obvious that $K$ is closed and convex, and the nonemptiness of $K$ is asserted by the selection theorem of Michael [9, Theorem 3.2]. To show that $f$ and $f^{*}$ are normal convex integrands, it suffices by [17, Theorem 3] to show that the multifunction $Q$ is measurable. The measurability of $Q$ follows in fact from lower semicontinuity, as has been observed by Castaing: for any closed set $W \subset R^{n}$, one has

$$
\{t \in T \mid Q(t) \cap W \neq \varnothing\}=\bigcup_{k=1}^{\infty} \bigcap_{m=1}^{\infty}\left\{t \in T \mid Q(t) \cap W_{k}^{m} \neq \varnothing\right\},
$$

where $W_{k}^{m}$ is the open set defined by

$$
W_{k}^{m}=\left\{x \in R^{n}|\exists z \in Q(t),| z|\leqq k,| x-z \mid<1 / m\right\} .
$$

The sets $\left\{t \in T \mid Q(t) \cap W_{k}^{m} \neq \varnothing\right\}$ are open, because $Q$ is lower semicontinuous, and hence $\{t \in T \mid Q(t) \cap W \neq \varnothing\}$ is a Borel set.

Fix any $\mu$ and $\theta$ as in the theorem. The functions $f(t, u(t))$ and $f^{*}(t, w(t))$ are summable with respect to $\theta$ for any $u \in K$ and $w=0$, so that $I_{f}$ on $L_{n}^{\infty}(T, \mathscr{T}, \theta)$ and $I_{f^{*}}$ on $L_{n}^{1}(T, \mathscr{T}, \theta)$ are convex functionals conjugate to each other. This implies in particular that

$$
I_{f^{*}}(d \mu / d \theta)=\sup \left\{\langle u, d \mu / d \theta\rangle_{\theta}-I_{f}(u) \mid u \in L_{n}^{\infty}(T, \mathscr{T} ; \theta)\right\},
$$

or, in other words,

$$
\begin{aligned}
\int_{T} f^{*}(t,(d \mu / d \theta)(t)) d \theta & =\sup _{u \in K^{\prime}} \int_{T}\langle u(t),(d \mu / d \theta)(t)\rangle d \theta \\
& \geqq \sup _{u \in K} \int_{T}\langle u(t),(d \mu / d \theta)(t)\rangle d \theta,
\end{aligned}
$$

where $K^{\prime}$ is the set of all bounded, measurable functions $u: T \rightarrow R^{n}$ such that $u(t) \in Q(t)$ for every $t$.

On the other hand, let $u_{0} \in K^{\prime}$ and $\alpha \in R^{1}$ be such that

$$
\alpha<\int_{T}\left\langle u_{0}(t),(d \mu / d \theta)(t)\right\rangle d \theta=\int_{T} u_{0} d \mu .
$$

We shall construct a $u \in K$ such that 


$$
\alpha<\int_{T}\langle u(t),(d \mu / d \theta)(t)\rangle d \theta=\int_{T} u d \mu,
$$

and this will prove the theorem.

Let $\beta>0$ be large enough that $\left|u_{0}(t)\right|<\beta$ for every $t$. Choose any $\varepsilon>0$ such that

$$
\alpha<\int_{T} u_{0} d \mu-2 \beta \varepsilon
$$

There exists by Lusin's Theorem a compact set $S \subset T$, such that $u_{0}$ is continuous relative to $S$, and

$$
\int_{S^{\prime}}|d \mu / d \theta| d \theta<\varepsilon, S^{\prime}=T \backslash S .
$$

Let $Q^{\prime}: T \rightarrow R^{n}$ be the multifunction defined by

$$
\begin{aligned}
Q^{\prime}(t) & =\left\{u_{0}(t)\right\} \quad \text { if } \quad t \in S, \\
& =\{z \in Q(t)|| z \mid<\beta\} \quad \text { if } \quad t \notin S .
\end{aligned}
$$

Then $Q^{\prime}(t)$ is for every $t$ a nonempty, convex set, $z \in Q^{\prime}(t)$ implies $|z|<\beta$, and the multifunction $\operatorname{cl} Q^{\prime}: t \rightarrow \operatorname{cl} Q^{\prime}(t)$ is lower semicontinuous. (To see the latter, let $U$ be any open set in $R^{n}$, and let $U_{\beta}$ be the open set consisting of all $x \in U$ such that $|x|<\beta$. Then

$$
\begin{aligned}
& \left\{t \in T \mid U \cap \operatorname{cl} Q^{\prime}(t) \neq \varnothing\right\} \\
= & \left\{t \in T \mid U \cap Q^{\prime}(t) \neq \varnothing\right\} \\
= & \left\{t \in T \mid Q(t) \cap U_{\beta} \neq \varnothing\right\} \backslash\left\{t \in S \mid u_{0}(t) \notin U\right\} .
\end{aligned}
$$

The latter set is open because $Q$ is lower semicontinuous, $S$ is closed, and $u_{0}$ is continuous relative to $S$.)

In view of the lower semicontinuity of $\operatorname{cl} Q^{\prime}$, there exists by Michael's Theorem [9] a function $u \in C_{n}(T)$ such that $u(t) \in \operatorname{cl} Q^{\prime}(t)$ for every $t$. In particular $u \in K,\|u\| \leqq \beta$, and $u$ agrees with $u_{0}$ on $S$. We then have

$$
\begin{aligned}
\int_{T} u d \mu & =\int_{T} u_{0} d \mu+\int_{S^{\prime}}\left(u-u_{0}\right) d \mu \\
& \geqq \int_{T} u_{0} d \mu-2 \beta \int_{S^{\prime}}|d \mu / d \theta| d \theta>\alpha
\end{aligned}
$$

by $(4.23)$ and $(4.24)$.

REMARK. Another refinement of Theorem 4, differing somewhat from Theorem 5, can be obtained by applying Theorem 6 to the epigraph multifunction

$$
Q: t \rightarrow \operatorname{epi} f_{t}=\left\{(x, \alpha) \in R^{n} \times R^{1} \mid \alpha \geqq f(t, x)\right\},
$$


provided that this multifunction is lower semicontinuous and $T$ is compact. The support function of epi $f_{t}$ can be expressed in terms of $f_{t}^{*}$ and its recession function $h$, as is well known, and this again yields formula (4.4).

5. Corollaries of the representation theorems. We now introduce some terminology for the purpose of stating a corollary of Theorem 5 about subgradients.

Let $N: T \rightarrow R^{n}$ be a multifunction such that $N(t)$ is for each $t$ a convex cone containing the origin. A measure $\nu \in M_{n}(T)$ will be called $N$-valued, if one has $(d \nu / d \theta)(t) \in N(t)$ except for a set of $\theta$ measure zero, where $\theta$ is a nonnegative measure in $M_{1}(T)$ with respect to which $\nu$ is absolutely continuous. (Note that this definition does not depend on the particular $\theta$, inasmuch as $N(t)$ is closed under multiplication by nonnegative scalars.) We shall be interested in the case where, for each $t, N(t)$ is the normal cone [15] to the convex set $\mathrm{cl} D(t)$ at a point $u(t) \in \mathrm{cl} D(t)$, in other words,

$$
N(t)=\left\{x^{*} \in R^{n} \mid\left\langle x-u(t), x^{*}\right\rangle \leqq 0, \forall x \in D(t)\right\} .
$$

CoROLlary 5A. Under the hypothesis of Theorem 5, a measure $\mu \in M_{n}(T)$ belongs to the subgradient set $\partial J_{f}(u)$, where $u \in C_{n}(T)$, if and only if $d \mu / d t$ satisfies (3.11'), $u(t)$ belongs to cl $D(t)$ for every $t$, and the singular component $\nu$ of $\mu$ is $N$-valued in the above sense, where $N(t)$ is the normal cone (5.1) to cl $D(t)$ at $u(t)$.

Proof. If $\partial J_{f}(u)$ is nonempty, then $u$ belongs to the convex set $E$, and consequently

$$
u(t) \in \operatorname{cl} D(t) \quad \text { for every } t,
$$

because the interior of $E$ is nonempty and consists of the functions satisfying (4.3). Since the inequalities

$$
\begin{gathered}
f^{*}(t,(d \mu / d t)(t)) \geqq\langle u(t),(d \mu / d t)(t)\rangle-f(t, u(t)), \\
h(t,(d \nu / d \theta)(t)) \geqq\langle u(t),(d \nu / d \theta)(t)\rangle,
\end{gathered}
$$

hold by (1.4) and (4.2) for any function $u$ satisfying (5.2), we see from formula (4.4) that a measure $\mu$ belongs to $\partial J_{f}(u)$, i.e., satisfies

$$
J_{f}^{*}(\mu)=\int_{T} u d \mu-J_{f}(u),
$$

if and only if (4.7) is true, (5.3) holds with equality except for a set of (dt-) measure zero, and (5.4) holds except for a set of $\theta$-measure zero. Of course, equality in (5.3) means that $(d u / d t)(t)$ is a subgradient of $f(t, \cdot)$ at $u(t)$. Equality in (5.4) means (for $u(t) \in \operatorname{cl} D(t)$ ) 
that $(d \nu / d \theta)(t)$ belongs to $N(t)$, inasmuch as $h(t, \cdot)$ is the support function of $D(t)$ by (4.2).

To state a corollary of Theorem 5 which characterizes the weak* closures of certain convex sets of measures, we recall that the recession cone of a convex set $C$ in $R^{n}$ is by definition the set of all vectors $y \in R^{n}$ such that $C+y \subset C[15, \S 8]$. (Some authors call this the asymptotic cone of $C$.)

COROLlaRY 5B. Let $Q: T \rightarrow R^{n}$ be a measurable multifunction such that $Q(t)$ is for each $t$ a nonempty, closed, convex set, and let $f_{t}=f(t, \cdot)$ be the support function of $Q(t)$. Assume that the hypothesis of Theorem 5 is satisfied by $f$ (which is a normal convex integrand). Let $W$ be the convex subset of $M_{n}(T)$ consisting of all the absolutely continuous measures $\mu$ such that

$$
\frac{d \mu}{d t}(t) \in Q(t) \text { for almost every } t .
$$

Then $J_{f}$ is the support function of $W$ on $C_{n}(T)$, and the following conditions on a measure $\mu \in M_{n}(T)$ are equivalent:

(a) $\mu$ belongs to the weak*-closure of $W$;

(b) (5.5) holds, and the singular component of $\mu$ is $N$-valued, where $N(t)$ is the recession cone of $Q(t)$;

(c) for every $u \in C_{n}(T)$, one has

$$
\int_{T} u d \mu \leqq \int_{T} f(t, u(t)) d t
$$

Proof. The normality of the convex integrand $f$ is equivalent to the measurability of $Q[17$, Theorem 3]. The conjugate integrand $f^{*}$ gives the indicator of $Q(t)$ for each $t$, and the recession function $h$ of $f^{*}$ gives the indicator of the recession cone $N(t)$ of $Q(t)$ for each $t[15, \S 14]$. The equivalence of (b) and (c) then follows from the formula for $J_{f}^{*}$ in Theorem 5. On the other hand, $I_{f}$ on $L_{n}^{\infty}(T)$ and $I_{f^{*}}$ on $L_{n}^{1}(T)$ are conjugate to each other by Theorem 2 (whose hypothesis is satisfied by an interior element $\bar{u}$ of the set $E$ in Theorem 5), so that

$$
\begin{aligned}
\int_{T} f(t, u(t)) d t & =\sup \left\{\int_{T}\left\langle u(t), u^{*}(t)\right\rangle d t-\int_{T} \delta_{Q(t)}\left(u^{*}(t)\right) d t\right\} \\
& =\sup \left\{\int_{T} u d \mu \mid \mu \in W\right\} .
\end{aligned}
$$

Thus $J_{f}$ is the support function of $W$, and this implies the equivalence of (a) and (c).

The recession cone $N(t)$ in Corollary $5 \mathrm{~B}$ is, of course, the polar 
of the effective domain $D(t)$ of the support function $f_{t}$ of $Q(t)$. The next corollary is an alternative form of Corollary $5 \mathrm{~B}$ in the case where $N(t)$ is independent of $t$ and contains no lines.

COROLlary 5C. The conclusions of Corollary 5B remain valid if, instead of assuming that $Q$ is measurable, and that the hypothesis of Theorem 5 is satisfied by $f$, one assumes the following: $T$ is compact (with no nonempty open sets of measure zero), the set $D(t)$ has a nonempty interior which is independent of $t$, and for each $x$ in this interior $f(t, x)$ is summable (measurable) function of $t$.

Proof. Since $D(t)$ has a fixed nonempty interior $D_{0}$, the multifunction $D: t \rightarrow D(t)$ is fully lower semicontinuous. Furthermore, $f$ is a normal convex integrand: condition (b) of the definition of normality in $\S 1$ is satisfied if $U$ is taken to be the set of all constant functions with values in $D_{0}^{\prime}$, where $D_{0}^{\prime}$ is any countable, dense subset of $D_{0}$. The hypothesis of Theorem 5 is thus satisfied, and Corollary $5 \mathrm{~B}$ can be applied.

These results enable us to describe a large class of weak* compact subsets of $M_{n}(T)$.

CoRollary 5D. Let the assumptions of either Corollary 5B or Corollary $5 C$ be satisfied, and let $\bar{W}$ denote the set of all measures $\mu \in M_{n}(T)$ for which condition (b) of Corollary $5 B$ holds. Then the set

$$
\bar{W}_{a, \alpha}=\left\{\mu \in \bar{W} \mid \int_{T} a d \mu \leqq \alpha\right\}
$$

is weak* compact in $M_{n}(T)$ for any $a \in C_{n}(T)$ satisfying

$$
-a(t) \in \operatorname{int} D(t) \quad \text { for every } t \in T,
$$

and any real number $\alpha$.

Proof. To derive this from the preceding corollaries, one need only verify that $\bar{W}_{a, \alpha}$ is bounded. Let $J$ denote the support function of $\bar{W}_{a, \alpha}$ on $C_{n}(T)$. Then $J \leqq J_{f}$. From Theorem 5 (whose hypothesis is satisfied under the assumption in $5 \mathrm{C}$ as well as $5 \mathrm{~B}$, as just demonstrated) we see that $J_{f}$ is finite on a neighborhood of $-a$. On the other hand, we have $J(\alpha) \leqq \alpha$ by hypothesis. The convexity of $J$ then implies that $J$ is finite on a neighborhood of the origin. Thus every linear functional on $M_{n}(T)$ corresponding to an element of $C_{n}(T)$ is bounded above on $\bar{W}_{a, \alpha}$, and hence $\bar{W}_{a, \alpha}$ is bounded.

REMARK. Corollary 5D generalizes a lemma developed by Olech $[13,14]$ for proving the existence of solutions to optimal control 
problems. If $T$ is metrizable in Corollary $5 \mathrm{D}$, then $\bar{W}_{a, \alpha}$ is sequentially weak* compact; hence in particular every sequence of absolutely continuous measures $\mu_{k} \in M_{n}(T)$ such that

$$
\frac{d \mu_{k}}{d t}(t) \in Q(t) \text { for almost every } t,
$$

$$
\int_{T} a(t) d \mu_{k} \leqq \alpha \text { for every } k,
$$

has a subsequence $\mu_{k_{i}}$ converging in the weak* topology to a measure $\mu$ which satisfies condition (b) of Corollary 5B. Olech's lemma [14, p. 515] states this fact in a slightly weaker form in the case where $T$ is the closure of a bounded Euclidean domain $G$, and the hypothesis of Corollary 5C is satisfied. (Olech's assumption (1.6) is equivalent to the existence of a constant function a satisfying our condition (5.9). He asserts only that the restrictions of the measures $\mu_{k_{i}}$ to $G$ converge to the restriction of $\mu$ to $G$ in the weak topology induced on $M_{n}(T)$ by the closure of the subspace of $C_{n}(T)$ consisting of the functions with compact support in $G$. However, his arguments imply convergence as described above, and they can even be extended to the case of arbitrary compact $T$, if sequences are replaced by nets. These arguments do not cover the case where int $D(t)$ is variable.)

We conclude by noting that Theorem 6 yields a result analogous to Corollary $5 \mathrm{~A}$.

COROLLARY 6A. Under the hypothesis of Theorem 6, the normal cone to the convex set $K$ at a point $u \in K$ is the set of all $N$-valued measures $\mu \in M_{n}(T)$, where $N(t)$ is for each $t$ the normal cone to $Q(t)$ at $u(t)$.

Added in proof. See [23] for additional references and results along the lines of this paper.

\section{REFERENCES}

1. N. Bourbaki, Espaces Vectoriels Topologiques, Hermann et Cie., Paris, 1953.

2. C. Castaing, Sur les multi-applications measurables, Thèse, Faculté des Sciences, Caën, 1967. This has partly been published in Rev. Franc. Inform. Rech. Operat., 1 (1967), 3-34.

3. C. Castaing, Un théorème de compacité faible dans $L_{E}^{1}$, Applications: sous-gradients et équations différentielles multivoques dans les espaces de Banach reflexifs non necessairement separables, Publication $\mathrm{N}^{0} 44$, Secretariat de Mathématiques, Université de Montpellier.

4. C. Castaing and M. Valadier, Équations différentielles dans les espaces localement convexes, C. R. Acad. Sci., Paris 266 (1968), 985-987.

5. C. Castaing, Quelques applications du theorème de Banach-Dieudonné a l'Intégration, Publication $N^{0}$ 67, Secrétariat de Mathématiques, Université de Montpellier. 
6. A. D. Ioffe and V. M. Tikhomirov, Duality of convex functions and extremum problems, Uspekhi Mat. Nauk., 23 (1968), 4-116.

7. - On the minimization of integral functionals, Funkt. Analiz, 3 (1969), 61-70.

8. K. Kuratowski and C. Ryll-Nardzewski, A general theorem on selectors, Bull. Polish Acad. Sci., 13 (1965), 397-411.

9. E. Michael, Continuous selections. I, Ann. of Math., 63 (1956), 361-382.

10. J. J. Moreau, Functionelles Convexes, mimeographed lecture notes, Séminaire sur les Équations aux Derivées Partielles, Collège de France, 1966-67.

11. — Sur la polaire d'une fonctionelle semicontinue supérieurment, C. R. Acad. Sci. Paris, 258 (1964), 1128-1130.

12. M. Nagumo, Über die gleichmässige Summierbarkeit und ihre Anwendung auf ein Variationsproblem, Japan J. Math., 6 (1929), 178-182.

13. C. Olech, Existence theorems for optimal problems with vector-valued cost function, Trans. Amer. Math. Soc., 136 (1969), 159-180.

14. - Exisience theorems for optimal control problems involving multiple integrals, J. Diff Eqs., 6 (1969), 512-526.

15. R. T. Rockafellar, Convex Analysis, Princeton Univ. Press, 1969.

16. - Integrals which are convex functionals, Pacific J. Math., 24 (1968), $525-539$.

17. - Measurable dependence of convex sets and functions on parameters, J. Math. Anal. Appl., 28 (1969), 4-25.

18. - Duality and stability in extremum problems involving convex functions, Pacific J. Math., 21 (1967), 167-187.

19. - Level sets and continuity of conjugate convex functions, Trans. Amer. Math Soc., 123 (1966), 46-63.

20. - Weak compactness of level sets of integral functionals, Proceedings of Troisième Colloque d'Analyse Fonctionelle (CBRM) (Liège, Sept. 1970), H. G. Garnir (editor), Vender (Louvain, Belgium), 1971.

21. M. Valadier, Contribution a l'analyse convexe, Thèse, University of Paris, 1970.

22. - Intégration de convexes fermés, notamment d'épigraphes. Inf-convolution continue, Institute de Recherche d'Informatique et d'Automatique, Aut. 7003.

23. R. T. Rockafellar, Convex integral functionals and duality, Contributions to Nonlinear Functional Analysis (E. Zarantonello, editor), Academic Press, 1971.

Received October 1969 and in revised form November 2, 1970. This work was supported in part by the Air Force Office of Scientific Research under grant no. AFAFOSR-1202-67B.

The author would like to thank A. D. Ioffe of the Moscow State University for some very helpful comments on an earlier version of this paper.

UNIVERSITY OF WASHINGTON 



\title{
PACIFIC JOURNAL OF MATHEMATICS
}

\author{
EDITORS
}

\author{
H. SAMELSON \\ Stanford University \\ Stanford, California 94305

\section{R. HOBBY} \\ University of Washington \\ Seattle, Washington 98105
}

J. DUGUNDJI

Department of Mathematics

University of Southern California

Los Angeles, California 90007

RICHARD ARENS

University of California

Los Angeles, California 90024

\section{ASSOCIATE EDITORS}
E. F. BECKENBACH
B. H. NeUmanN
F. WoLF
K. YoshidA

\section{SUPPORTING INSTITUTIONS}

\author{
UNIVERSITY OF BRITISH COLUMBIA \\ CALIFORNIA INSTITUTE OF TECHNOLOGY \\ UNIVERSITY OF CALIFORNIA \\ MONTANA STATE UNIVERSITY \\ UNIVERSITY OF NEVADA \\ NEW MEXICO STATE UNIVERSITY \\ OREGON STATE UNIVERSITY \\ UNIVERSITY OF OREGON \\ OSAKA UNIVERSITY \\ UNIVERSITY OF SOUTHERN CALIFORNIA
}

\author{
STANFORD UNIVERSITY \\ UNIVERSITY OF TOKYO \\ UNIVERSITY OF UTAH \\ WASHINGTON STATE UNIVERSITY \\ UNIVERSITY OF WASHINGTON \\ $* * * *{ }^{*}$
AMERICAN MATHEMATICAL SOCIETY
CHEVRON RESEARCH CORPORATION
NAVAL WEAPONS CENTER
}

The Supporting Institutions listed above contribute to the cost of publication of this Journal, but they are not owners or publishers and have no responsibility for its content or policies.

Mathematical papers intended for publication in the Pacific Journal of Mathematics should be in typed form or offset-reproduced, (not dittoed), double spaced with large margins. Underline Greek letters in red, German in green, and script in blue. The first paragraph or two must be capable of being used separately as a synopsis of the entire paper. The editorial "we" must not be used in the synopsis, and items of the bibliography should not be cited there unless absolutely necessary, in which case they must be identified by author and Journal, rather than by item number. Manuscripts, in duplicate if possible, may be sent to any one of the four editors. Please classify according to the scheme of Math. Rev. Index to Vol. 39. All other communications to the editors should be addressed to the managing editor, Richard Arens, University of California, Los Angeles, California, 90024.

50 reprints are provided free for each article; additional copies may be obtained at cost in multiples of 50 .

The Pacific Journal of Mathematics is published monthly. Effective with Volume 16 the price per volume (3 numbers) is $\$ 8.00$; single issues, $\$ 3.00$. Special price for current issues to individual faculty members of supporting institutions and to individual members of the American Mathematical Society: $\$ 4.00$ per volume; single issues $\$ 1.50$. Back numbers are available.

Subscriptions, orders for back numbers, and changes of address should be sent to Pacific Journal of Mathematics, 103 Highland Boulevard, Berkeley, California, 94708.

PUBLISHED BY PACIFIC JOURNAL OF MATHEMATICS, A NON-PROFIT CORPORATION

Printed at Kokusai Bunken Insatsusha (International Academic Printing Co., Ltd.), 270, 3chome Totsuka-cho, Shinjuku-ku, Tokyo 160, Japan. 


\section{Pacific Journal of Mathematics}

Vol. 39, No. $2 \quad$ June, 1971

Edward Arthur Bertram, Permutations as products of conjugate infinite

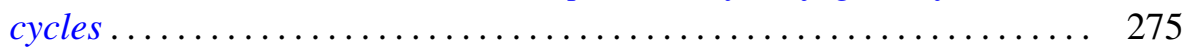

David Blair, Almost contact manifolds with Killing structure tensors ...... 285

Bruce Donald Calvert, Nonlinear equations of evolution ............. 293

Bohumil Cenkl and Giuliano Sorani, Cohomology groups associated with

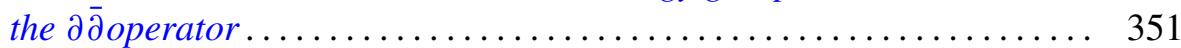

Martin Aaron Golubitsky and Bruce Lee Rothschild, Primitive subalgebras

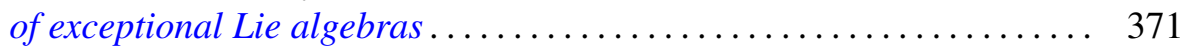

Thomas J. Jech, Two remarks on elementary embeddings of the universe ... 395

Harold H. Johnson, Conditions for isomorphism in partial differential equations........................................ 401

Solomon Leader, Measures on semilattices ..................... 407

Donald Steven Passman, Group rings satisfying a polynomial identity. II .......................................... 425

Ralph Tyrrell Rockafellar, Integrals which are convex functionals. II . . . . . 439

Stanisław Sławomir Świerczkowski, Cohomology of group germs and Lie

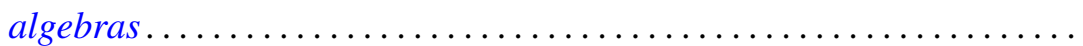

John Griggs Thompson, Nonsolvable finite groups all of whose local subgroups are solvable. III ............................ 483

Alan Curtiss Tucker, Matrix characterizations of circular-arc graphs .... 535 